\title{
Scattering theory for the Schrödinger-Debye System
}

\author{
Simão Correia and Filipe Oliveira
}

\begin{abstract}
We study the Schrödinger-Debye system over $\mathbb{R}^{d}$$$
\left\{\begin{array}{l}
i u_{t}+\frac{1}{2} \Delta u=u v \\
\mu v_{t}+v=\lambda|u|^{2}
\end{array}\right.
$$

and establish the global existence and scattering of small solutions for initial data in several function spaces in dimensions $d=2,3,4$. Moreover, in dimension $d=1$, we prove a Hayashi-Naumkin modified scattering result.
\end{abstract}

AMS Subject Classification 2010: 35Q55, 35Q60, 35B40.

Keywords: scattering theory; Schrödinger-Debye.

\section{Introduction}

In this work, we consider the initial value problem associated to the Schrödinger-Debye system

$$
\left\{\begin{array}{lc}
i u_{t}+\frac{1}{2} \Delta u=u v & (x, t) \in \mathbb{R}^{d} \times \mathbb{R}, d \leqslant 4 \\
\mu v_{t}+v=\lambda|u|^{2} & \mu>0, \lambda= \pm 1 \\
u(\cdot, 0)=u_{0}, & v(\cdot, 0)=v_{0}
\end{array}\right.
$$

where the function $u$ is complex-valued and $v$ is real. From a physical point of view, this model may describe the dynamics of an electromagnetic wave propagating through a nonresonant medium whose response time cannot be considered instantaneous (see Newell and Mahoney [15] and the references therein). When $\mu \rightarrow 0$, this response time vanishes and, in the limit case $\mu=0$, we obtain the classical cubic nonlinear Schrödinger equation

$$
i u_{t}+\frac{1}{2} \Delta u=\lambda|u|^{2} u .
$$

In this framework, one says that (NLS) is focusing (resp. defocusing) when $\lambda=-1$ (resp. $\lambda=1$ ).

The Cauchy problem (11) has been studied by several authors. Noticing that the second equation in (11) can be integrated in time, yielding

$$
v(t)=e^{-t / \mu} v_{0}+\frac{\lambda}{\mu} \int_{0}^{t} e^{-(s-t) / \mu}|u(s)|^{2} d s,
$$

one formally obtains

$$
i u_{t}+\frac{1}{2} \Delta u=\lambda u\left(e^{-t / \mu} v_{0}+\frac{\lambda}{\mu} \int_{0}^{t} e^{-(s-t) / \mu}|u(s)|^{2} d s\right),
$$


or, in integral form,

$$
u(t)=S(t) u_{0}+i \int_{0}^{t} S(t-s)\left(e^{-s / \mu} v_{0}+\frac{\lambda}{\mu} \int_{0}^{s} e^{-\left(s-s^{\prime}\right) / \mu}\left|u\left(s^{\prime}\right)\right|^{2} d s^{\prime}\right) u(s) d s,
$$

where $(S(t))_{t \in \mathbb{R}}=\left(e^{\frac{i}{2} \Delta t}\right)_{t \in \mathbb{R}}$ is the unitary Schrödinger group. We say that $(u, v)$ is a (weak) solution of the Cauchy problem (1) on if $(u, v)$ satisfies (443). For convenience, we synthethize several results shown in [2], [1, [4], [6], [5] in the following two theorems:

Theorem 1.1. Let $\left(u_{0}, v_{0}\right) \in H^{s}\left(\mathbb{R}^{d}\right) \times H^{s}\left(\mathbb{R}^{d}\right)$, where $d=1,2,3$ and $s=0,1$. Then there exists a unique solution to the Cauchy Problem (1), with

$$
(u, v) \in C\left([0,+\infty) ; H^{s}\left(\mathbb{R}^{d}\right) \times H^{s}\left(\mathbb{R}^{d}\right)\right) .
$$

Furthermore, if

$$
u_{0} \in \Sigma\left(\mathbb{R}^{d}\right):=\left\{f \in H^{1}\left(\mathbb{R}^{d}\right):|x| f \in L^{2}\left(\mathbb{R}^{d}\right)\right\},
$$

then $u \in C\left([0, \infty), \Sigma\left(\mathbb{R}^{d}\right)\right)$.

Theorem 1.2. Let $\left(u_{0}, v_{0}\right) \in H^{1}\left(\mathbb{R}^{4}\right) \times H^{1}\left(\mathbb{R}^{4}\right)$. Then, there exists $T=T\left(\left\|u_{0}\right\|_{H^{1}},\left\|v_{0}\right\|_{H^{1}}\right)$ and a unique solution to the initial value problem (1) in the time interval $[0, T]$ satisfying

$$
(u, v) \in C\left([0, T] ; H^{1}\left(\mathbb{R}^{4}\right) \times H^{1}\left(\mathbb{R}^{4}\right)\right),
$$

with

$$
\|u\|_{L^{\infty}\left((0, T), H_{x}^{1}\right)}+\|u\|_{L^{2}\left((0, T), W_{x}^{1,4}\right)}+\|v\|_{L^{\infty}\left((0, T), H_{x}^{1}\right)}<+\infty .
$$

Moreover, if $u_{0} \in \Sigma\left(\mathbb{R}^{4}\right)$, then $u \in C\left([0, T], \Sigma\left(\mathbb{R}^{4}\right)\right)$.

With respect to local existence results, we improve the existing theory in $d=4$ by showing

Theorem 1.3. Let $\left(u_{0}, v_{0}\right) \in L^{2}\left(\mathbb{R}^{4}\right) \times L^{2}\left(\mathbb{R}^{4}\right)$, $\left\|v_{0}\right\|_{L^{2}}$ sufficiently small. Then, there exists a unique maximal solution to the initial value problem (1) $(u, v)$ defined on $[0, T)$ such that

$$
u \in C\left([0, t], L^{2}\left(\mathbb{R}^{4}\right)\right) \cap L^{2}\left((0, t), L^{4}\left(\mathbb{R}^{4}\right)\right), v \in C\left([0, t] \cdot L^{2}\left(\mathbb{R}^{4}\right)\right), \quad t<T .
$$

If $T<\infty$, then

$$
\lim _{t \rightarrow T}\|u\|_{L^{2}\left((0, t), L^{4}\left(\mathbb{R}^{4}\right)\right)}=\infty .
$$

Furthermore, there exists $\epsilon>0$ such that, if $\left\|u_{0}\right\|_{L^{2}}<\epsilon$, then $T=\infty$ and

$$
\|u\|_{L^{\infty}\left((0, \infty), L^{2}\left(\mathbb{R}^{4}\right)\right.}+\|v\|_{L^{\infty}\left((0, \infty), L^{2}\left(\mathbb{R}^{4}\right)\right.}+\|u\|_{L^{2}\left((0, \infty), L^{4}\left(\mathbb{R}^{4}\right)\right)}<\infty .
$$

The system (1) exhibits a pseudo-scaling invariance: if $(u, v)$ is a solution of (1), then

$$
\left(u_{\mu^{*}}, v_{\mu^{*}}\right):=\left(\left(\frac{\mu^{*}}{\mu}\right)^{\frac{1}{2}} u\left(\left(\frac{\mu^{*}}{\mu}\right)^{\frac{1}{2}} x, \frac{\mu^{*}}{\mu} t\right), \frac{\mu^{*}}{\mu} v\left(\left(\frac{\mu^{*}}{\mu}\right)^{\frac{1}{2}} x, \frac{\mu^{*}}{\mu} t\right)\right)
$$

is the solution of (1) with $\mu$ replaced by $\mu^{*}$. Consequently, for convenience of notations, we will fix $\mu=1$ for the remainder of this work, even though our results remain valid for $\mu>0$.

Notice that (NLS) is invariant by (5). Considering that the Schrödinger-Debye system is a perturbation of (NLS), some authors use the definition of criticallity for the latter in the context of system (11): since

$$
\left\|u_{\mu^{*}}(t)\right\|_{L^{2}\left(\mathbb{R}^{d}\right)}=\left(\frac{\mu^{*}}{\mu}\right)^{\frac{2-d}{4}}\left\|u_{\mu}(t)\right\|_{L^{2}\left(\mathbb{R}^{d}\right)},\left\|\nabla u_{\mu^{*}}(t)\right\|_{L^{2}\left(\mathbb{R}^{d}\right)}=\left(\frac{\mu^{*}}{\mu}\right)^{\frac{4-d}{4}}\left\|\nabla u_{\mu}(t)\right\|_{L^{2}\left(\mathbb{R}^{d}\right)},
$$


one says that dimension $d=2$ is $L^{2}$-critical and dimension $d=4$ is $H^{1}$-critical. Note, however, that the local well-posedness results stated in Theorems 1.1 and 1.2 suggest that this analogy may be accurate. Furthermore, the conditional local well-posedness result stated in our Theorem 1.3 seems to indicate that it would be more adequate to consider the dimension $d=4$ as being $L^{2}$-critical.

The large time behaviour of small solutions is a central research topic in the field of nonlinear dispersive equations (see for instance [9], [10], [8], [16], 3]). Generally speaking, one expects small solutions to be globally defined and to scatter, that is, to tend, in a certain sense, to a solution of the corresponding linear problem. To the best of our knowledge, no such results regarding the Schrödinger-Debye system are currently available in the literature. In the present work, we will show several scattering results for small solutions of (11):

Theorem 1.4 (Scattering in dimension $d=4$ ). Let

$$
(X, Y) \in\left\{\left(L^{2}\left(\mathbb{R}^{4}\right), L^{2}\left(\mathbb{R}^{4}\right)\right),\left(H^{1}\left(\mathbb{R}^{4}\right), H^{1}\left(\mathbb{R}^{4}\right)\right),\left(\Sigma\left(\mathbb{R}^{4}\right), H^{1}\left(\mathbb{R}^{4}\right)\right)\right\} .
$$

There exists $\epsilon>0$ such that, if $\left(u_{0}, v_{0}\right) \in X \times Y$ satisfies $\left\|u_{0}\right\|_{X}+\left\|v_{0}\right\|_{Y}<\epsilon$, then the corresponding solution $(u, v)$ of (1) is global and scatters, that is, there exists $u_{+} \in X$ such that

$$
\left\|u(t)-S(t) u_{+}\right\|_{X} \rightarrow 0 \text { and }\|v(t)\|_{Y} \rightarrow 0, \quad t \rightarrow \infty .
$$

In the particular case $(X, Y)=\left(\Sigma\left(\mathbb{R}^{4}\right), H^{1}\left(\mathbb{R}^{4}\right)\right)$, the following decay estimate holds:

$$
\|u(t)\|_{L^{p}} \lesssim \frac{C\left(\left\|u_{0}\right\|_{\Sigma},\left\|v_{0}\right\|_{H^{1}}\right)}{t^{\left(2-\frac{4}{p}\right)}}, \quad t>0,2<p<4 .
$$

Theorem 1.5 (Scattering in dimensions $d=2,3)$. There exists $\delta>0$ such that, if $\left(u_{0}, v_{0}\right) \in$ $\Sigma\left(\mathbb{R}^{d}\right) \times H^{1}\left(\mathbb{R}^{d}\right), d=2,3$, satisfies $\left\|u_{0}\right\|_{H^{1}}+\left\|v_{0}\right\|_{H^{1}}<\delta$, then the corresponding solution $(u, v)$ of (11) scatters, that is, there exists $u_{+} \in \Sigma\left(\mathbb{R}^{d}\right)$ such that

$$
\left\|u(t)-S(t) u_{+}\right\|_{\Sigma} \rightarrow 0,\|v(t)\|_{H^{1}} \rightarrow 0, \quad t \rightarrow \infty .
$$

Furthermore,

$$
\|u(t)\|_{L^{p}} \lesssim \frac{C\left(\left\|u_{0}\right\|_{\Sigma},\left\|v_{0}\right\|_{H^{1}}\right)}{t^{d\left(\frac{1}{2}-\frac{1}{p}\right)}}, \quad t>0,2<p<2 d /(d-2)^{+} .
$$

In what concerns the dimension $d=1$, we observe that the Schrödinger Debye system exhibits a critical decay behaviour: indeed, if $u(t)=S(t) u_{0}$ and $v$ is defined by (3), then the linear decay of the unitary group implies, for large times, the critical decay of the nonlinear potential $v$ :

$$
\|v(t)\|_{L^{\infty}(\mathbb{R})} \sim \frac{1}{t} .
$$

As in the (NLS) case, this seriously compromises the chances of proving a classical result in dimension $d=1$. In this scenario, scattering results may be obtained provided that a phase correction in the Fourier space is introduced, the so-called modified scattering, i.e., denoting the Fourier transform by $\hat{\imath}$,

$$
\left\|e^{i \Psi(t)} S \widehat{(-t) u}(t)-\hat{u_{+}}\right\|_{X} \rightarrow 0, \quad t \rightarrow \infty,
$$

for some real-valued function $\Psi$ and some Banach space $X$ (see [11, [12]). With this in mind, following the ideas in [13, we show 
Theorem 1.6. There exists $\epsilon>0$ such that, if $\left(u_{0}, v_{0}\right) \in \Sigma(\mathbb{R}) \times H^{1}(\mathbb{R})$ satisfies $\left\|u_{0}\right\|_{H^{1}}+$ $\left\|v_{0}\right\|_{H^{1}}<\epsilon$, then the corresponding solution $(u, v)$ of (1) scatters up to a phase correction, that is, there exists (a unique) $u_{+} \in L^{2}(\mathbb{R})$ such that

$$
\left\|e^{i \Psi(t)} S \widehat{(-t) u}(t)-\hat{u_{+}}\right\|_{L^{2}} \rightarrow 0,\|v(t)\|_{L^{\infty}} \rightarrow 0, \quad t \rightarrow \infty
$$

where $\Psi(\xi, t)=\int_{1}^{t} \int_{1}^{s} \frac{1}{2 s^{\prime}} e^{-\left(s-s^{\prime}\right)}\left|\hat{f}\left(\frac{s}{s^{\prime}} \xi, s^{\prime}\right)\right|^{2} d s^{\prime} d s$. Also,

$$
\|u(t)\|_{L^{\infty}} \lesssim \frac{1}{t^{\frac{1}{2}}}, \quad t \rightarrow+\infty .
$$

Remark 1.7. As in [?], the proof of Theorem [1.6 will imply $\hat{u}_{+} \in L^{\infty}(\mathbb{R})$ and also an asymptotic expansion for the phase correction: for some $\Phi \in L^{\infty}(\mathbb{R})$, one has

$$
\left\|e^{i \Psi(t)}-\left|\hat{u}_{+}\right|^{2} \log t-\Phi\right\|_{\infty} \rightarrow 0, \quad t \rightarrow \infty .
$$

Notations. We denote the Fourier transform in the spatial variable by $\hat{\imath}$ If the Fourier transform is taken with respect to a particular variable $\xi$, we instead write $\mathcal{F}_{\xi}$. The spatial domain $\mathbb{R}^{d}$ will often be ommited. We abbreviate $L^{q}\left((0, T), L^{p}\left(\mathbb{R}^{d}\right)\right)$ as $L_{T}^{q} L_{x}^{r}$. The Sobolev space of elements in $L^{p}\left(\mathbb{R}^{d}\right)$ with derivatives in $L^{p}\left(\mathbb{R}^{d}\right)$ up to order $k$ is denoted by $W^{k, p}\left(\mathbb{R}^{d}\right)$. In the particular case where $p=2$, we set $H^{s}\left(\mathbb{R}^{d}\right):=W^{s, 2}\left(\mathbb{R}^{d}\right)$. We use the convention $2 /(d-2)^{+}=2 /(d-2)$, for $d \geqslant 3,2 /(d-2)^{+}=+\infty$, if $d=1,2$.

We end this introduction by recalling some Strichartz estimates for the unitary Schrödinger group, which we will use throughout the paper: we say that $(q, r)$ is an admissible pair in dimension $d$ if

$$
2 \leqslant r \leqslant 2 d /(d-2)^{+}, \quad \frac{2}{q}=d\left(\frac{1}{2}-\frac{1}{r}\right), \quad(q, r) \neq(2, \infty) .
$$

Then, for any couple of admissible pairs $(q, r)$ and $(\gamma, \rho)$, the following estimates hold:

$$
\begin{gathered}
\|S(t) u\|_{L_{t}^{q} L_{x}^{r}} \lesssim\|u\|_{L^{2}}, \quad t \in \mathbb{R}, \quad \text { (Homogeneous estimate) } \\
\left\|\int_{0}^{t} S(t-s) f(s) d s\right\|_{L_{t}^{q} L_{x}^{r}} \lesssim\|f\|_{L_{t}^{\gamma^{\prime}} L_{x}^{\rho^{\prime}}} \quad \text { (Inhomogeneous estimate). }
\end{gathered}
$$

\section{Scattering theory in dimension $d=4$}

In this section, we will establish scattering, global existence and decay of the solutions of the Schrödinger-Debye system (1) for small initial data in several spaces.

\section{1 $\quad L^{2}\left(\mathbb{R}^{4}\right) \times L^{2}\left(\mathbb{R}^{4}\right)$ theory}

First, we settle the local well-posedeness result for initial data in $L^{2}$.

Proof of Theorem 1.3. The proof of this result can be obtained by standard arguments (s ee [14]) from the a priori estimates

$$
\|u\|_{L_{t}^{2} L_{x}^{4}} \lesssim\left\|S(t) u_{0}\right\|_{L_{t}^{2} L_{x}^{4}}+\|u\|_{L_{t}^{2} L_{x}^{4}}^{3},
$$




$$
\|u\|_{L_{t}^{\infty} L_{x}^{2}} \lesssim\left\|u_{0}\right\|_{L^{2}}+\|u\|_{L_{t}^{2} L_{x}^{4}}^{3}
$$

and

$$
\|v\|_{L_{t}^{\infty} L_{x}^{2}} \leqslant\left\|v_{0}\right\|_{L^{2}}+\|u\|_{L_{t}^{2} L_{x}^{4}}^{2} .
$$

This last inequality is an immediate consequence of (3). The first one can be obtained from the Duhamel formula (44). Indeed, by the inhomogeneous Strichartz estimate,

$$
\begin{aligned}
\left\|\int_{0}^{t} S(t-s) e^{-s / \mu} v_{0} u(s) d s\right\|_{L_{t}^{2} L_{x}^{4}} & \lesssim\left\|e^{-t / \mu} v_{0} u\right\|_{L_{t}^{2} L_{x}^{4 / 3}} \\
& \lesssim\left\|e^{-t / \mu} u\right\|_{L_{t}^{2} L_{x}^{4}}\left\|v_{0}\right\|_{L_{t}^{\infty} L_{x}^{2}} \lesssim\|u\|_{L_{t}^{2} L_{x}^{4}}\left\|v_{0}\right\|_{L_{t}^{\infty} L_{x}^{2}} \lesssim\left\|v_{0}\right\|_{L^{2}}\|u\|_{L_{t}^{2} L_{x}^{4}}
\end{aligned}
$$

and

$$
\begin{aligned}
& \left\|\int_{0}^{t} S(t-s) \int_{0}^{s} e^{-\left(s-s^{\prime}\right) / \mu}\left|u\left(s^{\prime}\right)\right|^{2} u(s) d s^{\prime} d s\right\|_{L_{t}^{2} L_{x}^{4}}^{t} \\
& \lesssim\left\|\int_{0}^{t} e^{-\left(t-s^{\prime}\right) / \mu}\left|u\left(s^{\prime}\right)\right|^{2} u(t) d s^{\prime}\right\|_{L_{t}^{2} L_{x}^{4 / 3}} \lesssim\left\|\int_{0}^{t} e^{-\left(t-s^{\prime}\right) / \mu}\right\| u\left(s^{\prime}\right)\left\|_{L^{4}}^{2}\right\| u(t)\left\|_{L^{4}} d s^{\prime}\right\|_{L_{t}^{2}} \\
& \lesssim\|u\|_{L_{t}^{2} L_{x}^{4}} \int_{0}^{T} e^{-\left(T-s^{\prime}\right) / \mu}\left\|u\left(s^{\prime}\right)\right\|_{L^{4}}^{2} d s^{\prime} \leqslant\|u\|_{L_{t}^{2} L_{x}^{4}}^{3} .
\end{aligned}
$$

These estimates imply that

$$
\begin{aligned}
\|u\|_{L_{t}^{2} L_{x}^{4}} \lesssim & \left\|S(t) u_{0}\right\|_{L_{t}^{2} L_{x}^{4}}+\left\|\int_{0}^{t} S(t-s) e^{-s / \mu} v_{0} u(s) d s\right\|_{L_{L^{2} L_{x}^{4}}} \\
& +\left\|\int_{0}^{t} S(t-s) \int_{0}^{s} e^{-\left(s-s^{\prime}\right) / \mu}\left|u\left(s^{\prime}\right)\right|^{2} u(s) d s^{\prime} d s\right\|_{L_{t}^{2} L_{x}^{4}} \\
& \lesssim\left\|S(t) u_{0}\right\|_{L_{t}^{2} L_{x}^{4}}+\|u\|_{L_{t}^{2} L_{x}^{4}}\left\|v_{0}\right\|_{L^{2}}+\|u\|_{L_{t}^{2} L_{x}^{4}}^{3} .
\end{aligned}
$$

Now, since $\left\|v_{0}\right\|_{L^{2}}$ is taken arbitrarely small, (8) follows. Finally, the estimate (9) can be obtained by analogous computations.

As a direct consequence of the global well-posedness for small data, we prove Theorem 1.4 in the case $(X, Y)=\left(L^{2}\left(\mathbb{R}^{4}\right), L^{2}\left(\mathbb{R}^{4}\right)\right)$.

Proof of Theorem 1.4 for $(X . Y)=\left(L^{2}\left(\mathbb{R}^{4}\right), L^{2}\left(\mathbb{R}^{4}\right)\right)$. We set $f(t)=S(-t) u(t)$. Since

$$
\|u\|_{L^{2}\left((0, \infty), L_{x}^{4}\right)}<\infty
$$

we have

$$
\begin{aligned}
\left\|f(t)-f\left(t^{\prime}\right)\right\|_{L^{2}} & =\left\|S(t)\left(f(t)-f\left(t^{\prime}\right)\right)\right\|_{L^{2}} \\
& \lesssim\|u\|_{L^{2}\left(\left(t^{\prime}, t\right), L_{x}^{4}\right)}\left\|v_{0}\right\|_{L^{2}}+\|u\|_{L^{2}\left(\left(t^{\prime}, t\right), L_{x}^{4}\right)}^{3} \rightarrow 0, \quad t, t^{\prime} \rightarrow \infty
\end{aligned}
$$

Hence there exists $u_{+}:=\lim _{t \rightarrow \infty} S(-t) u(t) \in L^{2}\left(\mathbb{R}^{4}\right)$.

For any given $\delta>0$, pick $t^{\prime}$ so that $\|u\|_{L^{2}\left(\left(t^{\prime}, \infty\right), L_{x}^{4}\right)}<\delta$ and then choose $t$ large enough satisfying $e^{-\left(t-t^{\prime}\right)}\left\|v\left(t^{\prime}\right)\right\|_{L^{2}}<\delta$. Then

$$
\begin{aligned}
\|v(t)\|_{L^{2}} & \leqslant e^{-\left(t-t^{\prime}\right)}\left\|v\left(t^{\prime}\right)\right\|_{L^{2}}+\int_{t^{\prime}}^{t} e^{-(t-s)}\|u(s)\|_{L^{4}}^{2} d s \\
& \leqslant e^{-\left(t-t^{\prime}\right)}\left\|v\left(t^{\prime}\right)\right\|_{L^{2}}+\|u\|_{L^{2}\left(\left(t^{\prime}, t\right), L_{x}^{4}\right)}^{2}<2 \delta .
\end{aligned}
$$

This implies that $v(t) \rightarrow 0$ as $t \rightarrow \infty$, which concludes the proof. 


\section{$2.2 \quad H^{1}\left(\mathbb{R}^{4}\right) \times H^{1}\left(\mathbb{R}^{4}\right)$ theory}

We start with a simple remark regarding Theorem 1.1] from its proof (see 6]), it is standard to show the following blow-up alternative: putting

$$
T_{*}=\sup \left\{T>0:(u, v) \in C\left([0, T] ; H^{1}\left(\mathbb{R}^{4}\right) \times H^{1}\left(\mathbb{R}^{4}\right)\right)\right\},
$$

then

$$
T_{*}<+\infty \Rightarrow \lim _{T \rightarrow T_{*}}\left(\|\nabla u\|_{L_{T}^{\infty} L_{x}^{2}}+\|u\|_{L_{T}^{2} W_{x}^{1,4}}+\|v\|_{L_{T}^{\infty} H_{x}^{1}}\right)=+\infty .
$$

Notice that $\|u\|_{L^{2}}$ is conserved by the flow of the Schrödinger-Debye system. By (3),

$$
\|v\|_{L_{T}^{\infty} L_{x}^{2}} \leqslant\left\|v_{0}\right\|_{L^{2}}+\int_{0}^{T} e^{-(T-s)}\left\|u(s)^{2}\right\|_{L^{2}} d s \leqslant\left\|v_{0}\right\|_{L^{2}}+\|u\|_{L_{T}^{2} L_{x}^{4}}^{2}, \quad T<T_{*},
$$

and

$$
\begin{aligned}
\|\nabla v\|_{L_{T}^{\infty} L_{x}^{2}} & \leqslant\left\|\nabla v_{0}\right\|_{L^{2}}+\int_{0}^{T} e^{-(T-s)}\|u(s) \nabla u(s)\|_{L^{2}} d s \\
& \leqslant\left\|\nabla v_{0}\right\|_{L^{2}}+\int_{0}^{T}\|u(s)\|_{L^{4}}\|\nabla u(s)\|_{L^{4}} d s \\
& \leqslant\left\|\nabla v_{0}\right\|_{L^{2}}+\|\nabla u\|_{L_{T}^{2} L_{x}^{4}}\|u\|_{L_{T}^{2} L_{x}^{4}} .
\end{aligned}
$$

Therefore, we may express the blow-up alternative exclusively in terms of $u$ :

$$
T_{*}<+\infty \Rightarrow \lim _{T \rightarrow T_{*}}\left(\|\nabla u\|_{L_{T}^{\infty} L_{x}^{2}}+\|u\|_{L_{T}^{2} W_{x}^{1,4}}\right)=+\infty
$$

Using this fact, we will show the following result:

Proposition 2.1 (Global existence for small initial data in dimension $d=4$ ). There exists $\epsilon_{0}>0$ such that, if $\left\|u_{0}\right\|_{H^{1}}+\left\|v_{0}\right\|_{H^{1}}<\epsilon<\epsilon_{0}$, the solution $(u(t), v(t))$ given by Theorem 1.2 is global in time and

$$
\|u\|_{L^{\infty}\left((0, \infty), H_{x}^{1}\right)}+\|u\|_{L^{2}\left((0, \infty), W_{x}^{1,4}\right)} \leqslant 2 \epsilon .
$$

In order to show Proposition 2.1, we begin by proving some a priori estimates:

Lemma 2.2. Let $u_{0}, v_{0} \in H^{1}\left(\mathbb{R}^{4}\right)$ and $(u(t), v(t))$ the corresponding solution given by Theorem 1.2. Then, for any $T<T_{*}$,

$$
\|\nabla u\|_{L_{T}^{2} L_{x}^{4}}+\|\nabla u\|_{L_{T}^{\infty} L_{x}^{2}} \lesssim\left\|\nabla u_{0}\right\|_{L^{2}}+\left\|v_{0}\right\|_{L_{x}^{4}}\|u\|_{L_{T}^{\infty} H_{x}^{1}}+\left\|v_{0}\right\|_{H^{1}}\left\|u_{0}\right\|_{L^{2}}+\|\nabla u\|_{L_{T}^{2} L_{x}^{4}}\|u\|_{L_{T}^{2} L_{x}^{4}}^{2} .
$$

Proof. Differentiating (4),

$$
\begin{aligned}
\nabla u(t)=S(t) \nabla u_{0}+ & i \int_{0}^{t} S(t-s) e^{-s}\left(u(s) \nabla v_{0}+v_{0} \nabla u(s)\right) d s \\
& +2 i \lambda \int_{0}^{t} S(t-s) \int_{0}^{s} e^{-\left(s-s^{\prime}\right)} \operatorname{Re}\left(\nabla u\left(s^{\prime}\right) \overline{u\left(s^{\prime}\right)}\right) u(s) d s^{\prime} \\
& \quad+i \lambda \int_{0}^{t} S(t-s) \int_{0}^{s} e^{-\left(s-s^{\prime}\right)}\left|u\left(s^{\prime}\right)\right|^{2} \nabla u(s) d s^{\prime} d s .
\end{aligned}
$$


For $(q, r)=(2,4),(\infty, 2)$, we have $\left\|S(t) \nabla u_{0}\right\|_{L_{T}^{q} L_{x}^{r}} \lesssim\left\|\nabla u_{0}\right\|_{L^{2}}$ and

$$
\begin{aligned}
\| \int_{0}^{t} S(t-s) e^{-s}\left(u(s) \nabla v_{0}\right. & \left.+v_{0} \nabla u(s)\right) d s \|_{L_{T}^{q} L_{x}^{r}} \\
& \lesssim\left\|e^{-t}\left(\nabla v_{0} u+v_{0} \nabla u\right)\right\|_{L_{T}^{2} L_{x}^{4 / 3}} \lesssim\left\|v_{0}\right\|_{L^{2}}\|u\|_{L_{T}^{2} W_{x}^{1,4}}+\|u\|_{L_{T}^{\infty} L_{x}^{4}}\left\|v_{0}\right\|_{H^{1}} .
\end{aligned}
$$

Moreover,

$$
\begin{aligned}
\left\|\int_{0}^{t} S(t-s) \int_{0}^{s} e^{-\left(s-s^{\prime}\right)}\left|u\left(s^{\prime}\right)\right|^{2} \nabla u(s) d s^{\prime} d s\right\|_{L_{T}^{q} L_{x}^{r}} & \\
& \lesssim\left\|\int_{0}^{t} e^{-\left(t-s^{\prime}\right)}\left|u\left(s^{\prime}\right)\right|^{2} \nabla u(t) d s^{\prime}\right\|_{L_{T}^{2} L_{x}^{4 / 3}} \\
& \lesssim\left\|\int_{0}^{t} e^{-\left(t-s^{\prime}\right)}\right\| u\left(s^{\prime}\right)\left\|_{L^{4}}^{2}\right\| \nabla u(t)\left\|_{L^{4}} d s^{\prime}\right\|_{L_{T}^{2}} \lesssim\|u\|_{L_{T}^{2} L_{x}^{4}}^{2}\|\nabla u\|_{L_{T}^{2} L_{x}^{4}}
\end{aligned}
$$

and

$$
\begin{aligned}
& \left\|\int_{0}^{t} S(t-s) \int_{0}^{s} e^{-\left(s-s^{\prime}\right)} \nabla u\left(s^{\prime}\right) \overline{u\left(s^{\prime}\right)} u(s) d s^{\prime} d s\right\|_{L_{T}^{q} L_{x}^{r}}^{2} \\
& \lesssim\left\|\int_{0}^{s} e^{-\left(s-s^{\prime}\right)} \nabla u\left(s^{\prime}\right) \overline{u\left(s^{\prime}\right)} u(s) d s^{\prime}\right\|_{L_{T}^{2} L_{x}^{4 / 3}}^{2} \\
& \lesssim \int_{0}^{T}\left\|u(s)\left(\int_{0}^{s} e^{-\left(s-s^{\prime}\right)} \nabla u\left(s^{\prime}\right) \overline{u\left(s^{\prime}\right)} \| d s^{\prime}\right)\right\|_{L^{4 / 3}}^{2} d s \\
& \lesssim \int_{0}^{T}\|u(s)\|_{L^{4}}^{2}\left(\int_{0}^{s} e^{-\left(s-s^{\prime}\right)}\left\|\nabla u\left(s^{\prime}\right)\right\|_{L^{4}}\left\|u\left(s^{\prime}\right)\right\|_{L^{4}} d s^{\prime}\right)^{2} d s \\
& \lesssim\left(\int_{0}^{T}\left\|\nabla u\left(s^{\prime}\right)\right\|_{L^{4}}\left\|u\left(s^{\prime}\right)\right\|_{L^{4}} d s^{\prime}\right)^{2} \int_{0}^{T}\|u(s)\|_{L^{4}}^{2} d s \\
& \lesssim\|\nabla u\|_{L_{T}^{2} L_{x}^{4}}^{2}\|u\|_{L_{T}^{2} L_{x}^{4}}^{4},
\end{aligned}
$$

which concludes the proof.

Proof of Proposition 2.1. Putting $h(t)=\|u\|_{L_{T}^{\infty} H_{x}^{1}}+\|u\|_{L_{T}^{2} W_{x}^{1,4}}$, it follows from Lemma 2.2 and (8) that

$$
h(t) \leqslant C\left(\left\|u_{0}\right\|_{H^{1}}+h(t)\left\|v_{0}\right\|_{H^{1}}+h(t)^{3}\right),
$$

where $C$ is a positive constant. Therefore, choosing $\epsilon_{0}$ such that $C \epsilon_{0} \leqslant \frac{1}{2}$, we obtain that

$$
h(t) \leqslant 2 C\left(\left\|u_{0}\right\|_{H^{1}}+h(t)^{3}\right) .
$$

Thus, for $\left\|u_{0}\right\|_{H^{1}}<\epsilon$, a classical obstruction argument shows that $h(t)$ remains bounded by $2 \epsilon$. In view of the blow-up alternative (11), this implies that the solution is globally defined, which completes the proof of Proposition 2.1.

As in the $L^{2}$ case, the global well-posedness result implies scattering in $H^{1}\left(\mathbb{R}^{4}\right) \times H^{1}\left(\mathbb{R}^{4}\right)$, thus finishing the proof of Theorem 1.4 for $(X, Y)=\left(H^{1}\left(\mathbb{R}^{4}\right), H^{1}\left(\mathbb{R}^{4}\right)\right)$. 


\section{$2.3 \Sigma\left(\mathbb{R}^{4}\right) \times H^{1}\left(\mathbb{R}^{4}\right)$ theory}

Let us introduce the so-called vector field operator defined in $\Sigma\left(\mathbb{R}^{4}\right)=H^{1}\left(\mathbb{R}^{4}\right) \cap L^{2}(|x| d x)$ by

$$
\Gamma=x+i t \nabla .
$$

We recall that $\left[\Gamma, i \partial_{t}+\frac{1}{2} \Delta\right]=0$. We begin by showing

Lemma 2.3. In the conditions of Theorem [2.1, let $u_{0} \in \Sigma\left(\mathbb{R}^{4}\right)$. If $\left\|u_{0}\right\|_{\Sigma}+\left\|v_{0}\right\|_{H^{1}}<\epsilon$ small enough,

$$
\|\Gamma(u)\|_{L^{2}\left((0, \infty), L_{x}^{4}\right)}+\|\Gamma(u)\|_{L^{\infty}\left((0, \infty), L_{x}^{2}\right)} \lesssim\left\|u_{0}\right\|_{\Sigma}+\left\|v_{0}\right\|_{H^{1}} .
$$

Proof. From (11), putting $w=\Gamma(u)$,

$$
i w_{t}+\Delta w=\Gamma(u v)=x u v+i t(u \nabla v+v \nabla u)=v w+i t u \nabla v .
$$

By (3),

$$
\begin{aligned}
i w_{t}+\Delta w= & v w+i t e^{-t} u \nabla v_{0}+i t u \lambda \int_{0}^{t} e^{-(t-s)} \nabla|u(s)|^{2} d s \\
= & v w+i t e^{-t} u \nabla v_{0}+i u \lambda \int_{0}^{t}(t-s) e^{-(t-s)} \nabla|u(s)|^{2} d s \\
& +i u \lambda \int_{0}^{t} s e^{-(t-s)} \nabla|u(s)|^{2} d s \\
= & i t e^{-t} u \nabla v_{0}+v w+i u \lambda \int_{0}^{t}(t-s) e^{-(t-s)} \nabla|u(s)|^{2} d s \\
& +u \lambda \int_{0}^{t} e^{-(t-s)}\left(\Gamma\left(|u(s)|^{2}\right)-x|u(s)|^{2}\right) d s \\
= & i E(-t) u \nabla v_{0}+v w+i u \lambda \int_{0}^{t} E(-(t-s)) \nabla|u(s)|^{2} d s \\
& \quad+u \lambda \int_{0}^{t} e^{-(t-s)}(\bar{u} w-u \bar{w}) d s \\
:= & N_{1}(x, t)+N_{2}(x, t)+N_{3}(x, t)+N_{4}(x, t),
\end{aligned}
$$

where we have put $E(t)=-t e^{t}$ and used the identity

$$
i s \nabla(u \bar{u})=i s \nabla u \bar{u}+i s u \nabla \bar{u}=\bar{u}(\Gamma(u)-x u)+u(-\overline{\Gamma(u)}+x \bar{u})=\bar{u} w-u \bar{w} .
$$

In integral form, we obtain

$$
w(x, t)=S(t) w_{0}(x)+\sum_{i=1}^{4} \int_{0}^{t} S(t-s) N_{i}(x, s) d s .
$$

For $(q, r)=(2,4),(\infty, 2)$, the homogeneous Strichartz estimate yields

$$
\left\|S(t) w_{0}\right\|_{L_{T}^{q} L_{x}^{r}} \lesssim\left\|w_{0}\right\|_{L^{2}} \lesssim\left\|x u_{0}\right\|_{L^{2}}+\left\|u_{0}\right\|_{H^{1}} .
$$

Note that $E(-t)$ is bounded in $\left[0,+\infty\left[, \lim _{t \rightarrow \infty} E(-t)=0\right.\right.$ and $\int_{0}^{t} E(-(t-s)) d s=1-(t+1) e^{-t}$, which remains bounded as $t \rightarrow \infty$. Hence

$$
\left\|\int_{0}^{t} S(t-s) N_{1}(x, s) d s\right\|_{L_{T}^{q} L_{x}^{r}} \lesssim\left\|u \nabla v_{0}\right\|_{L_{T}^{2} L_{x}^{4 / 3}} \lesssim\|u\|_{L_{T}^{2} L_{x}^{4}}\|v\|_{L_{T}^{\infty} L_{x}^{2}}
$$


Also,

$$
\begin{aligned}
\left\|\int_{0}^{t} S(t-s) N_{2}(x, s) d s\right\|_{L_{T}^{q} L_{x}^{r}} & \lesssim\|v w\|_{L_{T}^{2} L_{x}^{4 / 3}} \lesssim\left(\int_{0}^{T}\|w(s)\|_{L^{4}}^{2}\|v(s)\|_{L^{2}}^{2} d s\right)^{\frac{1}{2}} \\
& \lesssim\|v\|_{L_{T}^{\infty} L_{x}^{2}} \mid w \|_{L_{T}^{2} L_{x}^{4} .}
\end{aligned}
$$

Following the steps of estimate (13),

$$
\left\|\int_{0}^{t} S(t-s) N_{3}(x, s) d s\right\|_{L_{T}^{q} L_{x}^{r}} \lesssim\|\nabla u\|_{L_{T}^{2} L_{x}^{4}}\|u\|_{L_{T}^{2} L_{x}^{4}}^{2} .
$$

Moreover, in view of (10),

$$
\left\|\int_{0}^{t} S(t-s) N_{4}(x, s) d s\right\|_{L_{T}^{q} L_{x}^{r}} \lesssim\|u\|_{L_{T}^{2} L_{x}^{4}}^{2}\|w\|_{L_{T}^{2} L_{x}^{4}} .
$$

We finally arrive at

$$
\begin{aligned}
\|\Gamma(u)\|_{L_{T}^{2} L_{x}^{4}}+\|\Gamma(u)\|_{L_{T}^{\infty} L_{x}^{2}} & \lesssim\left\|x u_{0}\right\|_{L^{2}}+\left\|u_{0}\right\|_{H^{1}}+\|u\|_{L_{T}^{2} L_{x}^{4}}\|v\|_{L_{T}^{\infty} L_{x}^{2}}+\|v\|_{L_{T}^{\infty} L_{x}^{2}}\|\Gamma(u)\|_{L_{T}^{2} L_{x}^{4}} \\
& +\|\nabla u\|_{L_{T}^{2} L_{x}^{4}}\|u\|_{L_{T}^{2} L_{x}^{4}}^{2}+\|u\|_{L_{T}^{2} L_{x}^{4}}^{2}\|\Gamma(u)\|_{L_{T}^{2} L_{x}^{4}} .
\end{aligned}
$$

In view of Proposition 2.1, choosing $\left(u_{0}, v_{0}\right)$ small enough such that

$$
\|u\|_{L^{2}\left((0, \infty), L_{x}^{4}\right)}^{2}+\|v\|_{L^{\infty}\left((0, \infty), L_{x}^{2}\right)}<\frac{1}{2},
$$

we obtain, as stated in Lemma 2.3

$$
\|\Gamma(u)\|_{L_{T}^{2} L_{x}^{4}}+\|\Gamma(u)\|_{L_{T}^{\infty} L_{x}^{2}} \lesssim\left\|u_{0}\right\|_{\Sigma}+\left\|v_{0}\right\|_{H^{1}}, \quad T>0 .
$$

Proof of Theorem 1.4 in the case $(X, Y)=\left(\Sigma\left(\mathbb{R}^{4}\right), H^{1}\left(\mathbb{R}^{4}\right)\right)$. The convergence (6) follows easily from the previous lemma. The decay (17) is obtained by Gagliardo-Nirenberg's inequality: indeed, writing $z=e^{\frac{-i|x|^{2}}{2 t}} u$, we have $\Gamma(u)=i t e^{\frac{i|x|^{2}}{2 t}} \nabla z$. Then, by the previous lemma,

$$
\|\nabla z(t)\|_{L^{2}}=\frac{1}{t}\|\Gamma(u)(t)\|_{L^{2}} \lesssim \frac{\left\|u_{0}\right\|_{\Sigma}+\left\|v_{0}\right\|_{H^{1}}}{t} .
$$

Hence

$$
\|u(t)\|_{L^{p}}=\|z(t)\|_{L^{p}} \lesssim\|z(t)\|_{L^{2}}^{1-d\left(\frac{1}{2}-\frac{1}{p}\right)}\|\nabla z(t)\|_{L^{2}}^{d\left(\frac{1}{2}-\frac{1}{p}\right)} \lesssim \frac{\left\|u_{0}\right\|_{\Sigma}+\left\|v_{0}\right\|_{H^{1}}}{t^{d\left(\frac{1}{2}-\frac{1}{p}\right)}} .
$$

\section{Scattering theory in dimensions $d=2,3$}

In this section, we prove Theorem 1.5. We start with the three-dimensional case. 


\subsection{Proof of Theorem 1.5 in dimension $d=3$}

Contrarely to the fourth dimensional case studied in the previous section, in order to obtain a scattering result in dimension 3, we must first prove some time decay for the solutions of (11):

Proposition 3.1. Let $\left(u_{0}, v_{0}\right) \in \Sigma\left(\mathbb{R}^{3}\right) \times H^{1}\left(\mathbb{R}^{3}\right)$ and $(u(t), v(t))$ the corresponding solution to (1) given by Theorem 1.1. Then, there exists $\delta>0$ such that, if $\left\|u_{0}\right\|_{\Sigma}+\left\|v_{0}\right\|_{L^{2}}<\delta$,

$$
\|u\|_{L^{\infty}\left((0, \infty), H_{x}^{1}\right)}+\|u\|_{L^{\frac{8}{3}}\left((0, \infty), W_{x}^{1,4}\right)}<2 \delta
$$

and, for all $t>0$,

$$
\|u(t)\|_{L^{4}} \lesssim \frac{1}{t^{\frac{3}{4}}}
$$

Before proving this result, we will need the following two preliminary lemmas:

Lemma 3.2. Let $\left(u_{0}, v_{0}\right) \in H^{1}\left(\mathbb{R}^{3}\right) \times H^{1}\left(\mathbb{R}^{3}\right)$ and $(u(t), v(t))$ the corresponding solution to (1) given by Theorem 1.1. Then, there exists $\epsilon>0$ such that, whenever $\left\|u_{0}\right\|_{H^{1}}+\left\|v_{0}\right\|_{H^{1}}<\delta, \delta$ small, and, for some $T>1$,

$$
\|u\|_{T}:=\sup _{0<t<T}\left\{t^{\alpha}\|u(t)\|_{L_{x}^{4}}\right\} \leqslant \epsilon, \quad \alpha>\frac{5}{8},
$$

then

$$
\|u\|_{L_{T}^{\infty} H_{x}^{1}}+\|u\|_{L_{T}^{\frac{8}{3}} W_{x}^{1,4}}<2 \delta .
$$

Proof. Using the Duhamel formula (4), we begin by estimating $u$ in the Strichartz norms $L_{T}^{q} L_{x}^{r}$, $(q, r)=\left(\frac{8}{3}, 4\right),(\infty, 2)$ :

$$
\left\|\int_{0}^{t} S(t-s) e^{-s} v_{0} u(s) d s\right\|_{L_{T}^{q} L_{x}^{r}} \lesssim\left\|e^{-t} v_{0} u(t)\right\|_{L_{T}^{\frac{8}{5}} L_{x}^{4 / 3}} \lesssim\|\| e^{-t} u\left\|_{L^{4}}\right\| v_{0}\left\|_{L^{2}}\right\|_{L_{T}^{\frac{8}{5}}}
$$

and

$$
\left\|\int_{0}^{t} S(t-s) e^{-s} v_{0} u(s) d s\right\|_{L_{T}^{q} L_{x}^{r}} \lesssim\left\|v_{0}\right\|_{L^{2}}\|u\|_{L_{T}^{\frac{8}{3}} L_{x}^{4}},
$$

where we have used the Hölder inequality. Also,

$$
\begin{aligned}
& \left\|\int_{0}^{t} S(t-s) \int_{0}^{s} e^{-\left(s-s^{\prime}\right)}\left|u\left(s^{\prime}\right)\right|^{2} u(s) d s^{\prime} d s\right\|_{L_{T}^{q} L_{x}^{r}} \\
& \lesssim\left\|\int_{0}^{t} e^{-\left(t-s^{\prime}\right)}\right\| u\left(s^{\prime}\right)\left\|_{L^{4}}^{2}\right\| u(t)\left\|_{L^{4}} d s^{\prime}\right\|_{L_{T}^{\frac{8}{5}} L_{x}^{4}} \\
& \lesssim\|u\|_{L_{T}^{\frac{8}{3}} L_{x}^{4}}\left\|\int_{0}^{t} e^{-\left(t-s^{\prime}\right)}\right\| u\left(s^{\prime}\right)\left\|_{L^{4}}^{2} d s^{\prime}\right\|_{L_{T}^{4}}
\end{aligned}
$$

We now split the time interval $[0 ; T]$ :

$$
\left\|\int_{0}^{t} e^{-\left(t-s^{\prime}\right)}\right\| u\left(s^{\prime}\right)\left\|_{L^{4}}^{2} d s^{\prime}\right\|_{L^{4}(0,1)} \lesssim\|u\|_{L_{T}^{\infty} L_{x}^{4}}^{2} \lesssim\|u\|_{L_{T}^{\infty} H_{x}^{1}}^{2}
$$

and

$$
\left\|\int_{0}^{t} e^{-\left(t-s^{\prime}\right)}\right\| u\left(s^{\prime}\right)\left\|_{L^{4}}^{2} d s^{\prime}\right\|_{L^{4}(1, T)} \leqslant\left\|\int_{0}^{t / 2} e^{-\left(t-s^{\prime}\right)}\right\| u\left(s^{\prime}\right)\left\|_{L^{4}}^{2} d s^{\prime}\right\|_{L^{4}(1, T)}
$$




$$
\begin{gathered}
\quad+\left\|\int_{t / 2}^{t} e^{-\left(t-s^{\prime}\right)}\right\| u\left(s^{\prime}\right)\left\|_{L^{4}}^{2} d s^{\prime}\right\|_{L^{4}(1, T)} \\
\leqslant\|u\|_{L_{T}^{\infty} H_{x}^{1}}+\|u\|_{T}^{2}\left\|\int_{t / 2}^{t} \frac{1}{s^{\prime 2 \alpha}} d s^{\prime}\right\|_{L^{4}(1, T)} \\
\leqslant\|u\|_{L_{T}^{\infty} H_{x}^{1}}+\|u\|_{T}^{2}\left(\int_{1}^{T} \frac{1}{t^{4(2 \alpha-1)}} d t\right)^{4}
\end{gathered}
$$

and this last integral is bounded for $\alpha>\frac{5}{8}$. Hence,

$$
\left\|\int_{0}^{t} S(t-s) \int_{0}^{s} e^{-\left(s-s^{\prime}\right)}\left|u\left(s^{\prime}\right)\right|^{2} u(s) d s^{\prime} d s\right\|_{L_{T}^{q} L_{x}^{r}} \lesssim\|u\|_{L_{T}^{\frac{8}{3}} L_{x}^{4}}\left(\|u\|_{L_{T}^{\infty} H_{x}^{1}}^{2}+\|u\|_{T}^{2}\right) .
$$

Combining this last inequality with (15), we obtain

$$
\|u\|_{L_{T}^{q} L_{x}^{r}} \lesssim\left\|u_{0}\right\|_{L^{2}}+\|u\|_{L_{T}^{\frac{8}{3}} L_{x}^{4}}\left(\left\|v_{0}\right\|_{L^{2}}+\|u\|_{L_{T}^{\infty} H_{x}^{1}}^{2}+\|u\|_{T}^{2}\right)
$$

Next, in view of (12), we estimate $\|\nabla u\|_{L_{T}^{q} L_{x}^{r}}$. As in (15),

$$
\left\|\int_{0}^{t} S(t-s) e^{-s}\left(u(s) \nabla v_{0}+v_{0} \nabla u(s)\right) d s\right\|_{L_{T}^{q} L_{x}^{r}} \lesssim\left\|\nabla v_{0}\right\|_{L^{2}}\|u\|_{L_{T}^{\frac{8}{3}} L_{x}^{4}}+\left\|v_{0}\right\|_{L^{2}}\|\nabla u\|_{L_{T}^{\frac{8}{3}} L_{x}^{4}} .
$$

Also, as in (16),

$$
\left\|\int_{0}^{t} S(t-s) \int_{0}^{s} e^{-\left(s-s^{\prime}\right)}\left|u\left(s^{\prime}\right)\right|^{2} \nabla u(s) d s^{\prime} d s\right\|_{L_{T}^{q} L_{r}^{4}} \lesssim\|\nabla u\|_{L_{T}^{\frac{8}{3}} L_{x}^{4}}\left(\|u\|_{L_{T}^{\infty} H_{x}^{1}}^{2}+\|u\|_{T}^{2}\right) .
$$

Furthermore, by Hölder,

$$
\begin{aligned}
& \left\|\int_{0}^{t} S(t-s) \int_{0}^{s} e^{-\left(s-s^{\prime}\right)} \operatorname{Re}\left(\nabla u\left(s^{\prime}\right) \overline{u\left(s^{\prime}\right)}\right) u(s) d s^{\prime}\right\|_{L_{T}^{q} L_{x}^{r}} \\
& \quad \lesssim\|\| u(t)\left\|_{L^{4}} \int_{0}^{t} e^{-\left(t-s^{\prime}\right)}\right\| \nabla u\left(s^{\prime}\right)\left\|_{L^{4}}\right\| u\left(s^{\prime}\right)\left\|_{L^{4}} d s^{\prime}\right\|_{L_{T}^{\frac{8}{5}}} \\
& \lesssim\|\nabla u\|_{L_{T}^{\frac{8}{3}} L_{x}^{4}}\|\| u(t)\left\|_{L^{4}}\left(\int_{0}^{t} e^{-\frac{8}{5}\left(t-s^{\prime}\right)}\left\|u\left(s^{\prime}\right)\right\|_{L^{4}}^{\frac{8}{5}} d s^{\prime}\right)^{\frac{5}{8}}\right\|_{L_{T}^{\frac{8}{5}}} .
\end{aligned}
$$

Now,

$$
\begin{gathered}
\|\| u(t)\left\|_{L^{4}}\left(\int_{0}^{t} e^{-\frac{8}{5}\left(t-s^{\prime}\right)}\left\|u\left(s^{\prime}\right)\right\|_{L^{4}}^{\frac{8}{5}} d s^{\prime}\right)^{\frac{5}{8}}\right\|_{L^{\frac{8}{5}}(0,1)} \\
\lesssim\|u\|_{L_{T}^{\frac{8}{3}} L_{x}^{4}}\left\|\left(\int_{0}^{t} e^{-\frac{8}{5}\left(t-s^{\prime}\right)}\left\|u\left(s^{\prime}\right)\right\|_{L^{4}}^{\frac{8}{5}} d s^{\prime}\right)^{\frac{5}{8}}\right\|_{L^{4}(0,1)} \lesssim\|u\|_{L_{T}^{\frac{8}{3}} L_{x}^{4}}\|u\|_{L_{T}^{\infty} H_{x}^{1}}
\end{gathered}
$$

and, similarly

$$
\|\| u(t)\left\|_{L^{4}}\left(\int_{0}^{\frac{t}{2}} e^{-\frac{8}{5}\left(t-s^{\prime}\right)}\left\|u\left(s^{\prime}\right)\right\|_{L^{4}}^{\frac{8}{5}} d s^{\prime}\right)^{\frac{5}{8}}\right\|_{L^{\frac{8}{5}}(0,1)} \lesssim\|u\|_{L_{T}^{\frac{8}{3}} L_{x}^{4}}\|u\|_{L_{T}^{\infty} H_{x}^{1}} .
$$

Finally, using the time decay,

$$
\|\| u(t)\left\|_{L^{4}}\left(\int_{\frac{t}{2}}^{t} e^{-\frac{8}{5}\left(t-s^{\prime}\right)}\left\|u\left(s^{\prime}\right)\right\|_{L^{4}}^{\frac{8}{5}} d s^{\prime}\right)^{\frac{5}{8}}\right\|_{L^{\frac{8}{5}(1, T)}} \lesssim\|u\|_{T}^{2} \int_{1}^{T} \frac{1}{s^{\frac{8 \alpha}{5}}} \frac{1}{s^{\frac{8 \alpha}{5}}-1} d s \lesssim\|u\|_{T}^{2}
$$


for $\alpha>\frac{5}{8}$. Hence

$$
\begin{gathered}
\|\nabla u\|_{L_{T}^{q} L_{x}^{r}} \lesssim\left\|\nabla u_{0}\right\|_{L^{2}}+\left\|\nabla v_{0}\right\|_{L^{2}}\|u\|_{L_{T}^{\frac{8}{3}} L_{x}^{4}}+\left\|v_{0}\right\|_{L^{2}}\|\nabla u\|_{L_{T}^{\frac{8}{3}} L_{x}^{4}}+ \\
\|\nabla u\|_{L_{T}^{\frac{8}{3}} L_{x}^{4}}\left(\|u\|_{L_{T}^{\infty} H^{1}}^{2}+\|u\|_{T}^{2}\right)+\|\nabla u\|_{L_{T}^{\frac{8}{3}} L_{x}^{4}}\left(\|u\|_{L_{x}^{\infty} H_{x}^{1}}^{2}+\|u\|_{T}^{2}\right) .
\end{gathered}
$$

Combining this inequality with (17) and putting $h(T)=\|u\|_{L^{\infty}\left((0, T), H_{x}^{1}\right)}+\|u\|_{L^{\frac{8}{3}}\left((0, T), W_{x}^{1,4}\right)}$ we obtain, for small $\delta$ and $\epsilon$,

$$
h(t) \lesssim \delta+h(t)^{3},
$$

and the Lemma is proved by using once again an obstruction argument.

Lemma 3.3. In the conditions of Lemma 3.2, if additionally $x u_{0} \in L^{2}\left(\mathbb{R}^{3}\right)$ and $\left\|u_{0}\right\|_{\Sigma}+\left\|v_{0}\right\|_{L^{2}}<$ $\delta$, then, for all $t \leqslant T$,

$$
\|u(t)\|_{L^{4}} \lesssim \frac{\delta}{t^{\frac{3}{4}}} .
$$

Proof. Arguing as in the proof of Theorem 1.4 in the case $(X, Y)=\left(\Sigma\left(\mathbb{R}^{4}\right), H^{1}\left(\mathbb{R}^{4}\right)\right)$, we only need to show that $\|\Gamma(u)\|_{L_{T}^{\infty} L_{x}^{2}}<\delta$, which can be obtained from an a priori estimate as in Lemma 2.3 . This can be easily obtained by using similar computations as in the proof of Lemma 3.2 to estimate the $L_{T}^{\infty} L_{x}^{2}$ norm of the right-hand-side of (14).

We are now able to show Theorem 1.5 in the case $d=3$ :

Proof of Theorem 3.1. Let $\delta>0$ and $\left(u_{0}, v_{0}\right)$ such that $\left\|u_{0}\right\|_{\Sigma}+\left\|v_{0}\right\|_{H^{1}}<\delta$. Define $T^{*}=$ $\sup \left\{T:\|u\|_{T}<\epsilon\right\}$, for a fixed $\epsilon>0$ small enough as in Lemma 3.2. Our goal is to establish that $T^{*}=+\infty$ provided that $\delta>0$ is small enough. By contradiction, we assume that $T^{*}<+\infty$. From the local well-posedness result, we have, for $\delta$ small enough,

$$
\|u\|_{T=1} \lesssim\|u\|_{L^{\infty}\left((0,1), L_{x}^{4}\right)} \lesssim\|u\|_{L^{\infty}\left((0,1), H_{x}^{1}\right)} \lesssim\left\|u_{0}\right\|_{H^{1}}+\left\|v_{0}\right\|_{H^{1}} \lesssim \delta
$$

which implies that $\|u\|_{T=1}<\epsilon$, hence $T^{*}>1$.

On the other hand, from Lemma 3.3, $T^{*^{\frac{3}{4}}}\left\|u\left(T^{*}\right)\right\|_{L^{4}} \lesssim \delta$. Also, by definition of $T^{*}$ and by continuity of $t \rightarrow u(\cdot, t) \in L^{4}, T^{* \alpha}\left\|u\left(T^{*}\right)\right\|_{L^{4}}=\epsilon$. Hence, $\delta \gtrsim T^{* \frac{3}{4}-\alpha} \gtrsim 1$ for $\frac{5}{8}<\alpha<\frac{3}{4}$, which is absurd for small $\delta$, and the proof of Theorem 1.5 for $d=3$ follows.

\subsection{Proof of Theorem 1.5 in dimension $d=2$}

The proof of the scattering result in dimension $d=2$ follows the lines of the three dimensional case. However, the optimal linear asymptotic decay, for $u_{0} \in \Sigma\left(\mathbb{R}^{2}\right)$, reads

$$
\left\|S(t) u_{0}\right\|_{L^{4}} \sim \frac{1}{t^{\frac{1}{2}}},
$$

hence we must prove a version of Lemma 3.2 valid for $\alpha=\frac{1}{2}$ : 
Lemma 3.4. Let $\left(u_{0}, v_{0}\right) \in H^{1}\left(\mathbb{R}^{2}\right) \times H^{1}\left(\mathbb{R}^{2}\right)$ and $(u(t), v(t))$ the corresponding solution to (1) given by Theorem 1.1. Then, there exists $\epsilon>0$ such that, whenever $\left\|u_{0}\right\|_{H^{1}}+\left\|v_{0}\right\|_{H^{1}}<\delta, \delta$ small, and, for some $T>1$,

$$
\|u\|_{T}:=\sup _{0<t<T}\left\{t^{\alpha}\|u(t)\|_{L_{x}^{4}}\right\} \leqslant \epsilon, \quad \alpha>\frac{3}{8},
$$

then

$$
\|u\|_{L_{T}^{\infty} H_{x}^{1}}+\|u\|_{L_{T}^{4} W_{x}^{1,4}}<2 \delta .
$$

Unfortunately, the splitting of the time interval $[0, t]$ into $[0, t / 2]$ and $[t / 2 ; t]$ will no longer provide, in this dimension, adequate estimates for the $L_{T}^{q} L_{x}^{r}$ Strichartz norms $(q, r)=(4,4),(\infty, 2)$ of the cubic integrals

$$
\nabla^{(i)} \int_{0}^{t} S(t-s) \int_{0}^{s} e^{-\left(s-s^{\prime}\right)}\left|u\left(s^{\prime}\right)\right|^{2} u(s) d s^{\prime} d s, \quad i=0,1 .
$$

Hence, using a different technique, we will show the following Lemma, from which Lemma 3.4 and Theorem 1.5 follow:

Lemma 3.5. Let $\alpha>\frac{3}{8}$ and $(q, r)=(4,4),(\infty, 2)$. Then

$$
\left\|\int_{0}^{t} S(t-s) \int_{0}^{s} e^{-\left(s-s^{\prime}\right)}\left|u\left(s^{\prime}\right)\right|^{2} u(s) d s^{\prime} d s\right\|_{L_{T}^{q} L_{x}^{r}} \lesssim\|u\|_{L_{T}^{4} L_{x}^{4}}\|u\|_{T}^{2}
$$

and

$$
\begin{aligned}
& \left\|\nabla \int_{0}^{t} S(t-s) \int_{0}^{s} e^{-\left(s-s^{\prime}\right)}\left|u\left(s^{\prime}\right)\right|^{2} u(s) d s^{\prime} d s\right\|_{L_{T}^{q} L_{x}^{r}} \\
& \quad \lesssim\|u\|_{L_{T}^{4} W_{x}^{1,4}}\|u\|_{L_{T}^{\infty} H_{x}^{1}}^{2}+\|\nabla u\|_{L_{T}^{4} L_{x}^{4}}\|u\|_{T}^{2} .
\end{aligned}
$$

Proof. By the inhomogeneous Strichartz estimate,

$$
\left\|\nabla^{(i)} \int_{0}^{t} S(t-s) \int_{0}^{s} e^{-\left(s-s^{\prime}\right)}\left|u\left(s^{\prime}\right)\right|^{2} u(s) d s^{\prime} d s\right\|_{L_{T}^{q} L_{x}^{r}} \lesssim\left\|\nabla^{(i)} \int_{0}^{s} e^{-\left(s-s^{\prime}\right)}\left|u\left(s^{\prime}\right)\right|^{2} u(s) d s^{\prime}\right\|_{L_{T}^{\frac{4}{3}} L_{x}^{\frac{4}{3}}} .
$$

For $i=0$,

$$
\begin{gathered}
\left\|\int_{0}^{s} e^{-\left(s-s^{\prime}\right)}\left|u\left(s^{\prime}\right)\right|^{2} u(s) d s^{\prime}\right\|_{L_{T}^{\frac{4}{3}} L_{x}^{\frac{4}{3}}} \leqslant\left\|\int_{0}^{s} e^{-\left(s-s^{\prime}\right)}\right\| u\left(s^{\prime}\right)\left\|_{L^{4}}^{2}\right\| u(s)\left\|_{L^{4}} d s^{\prime}\right\|_{L_{T}^{\frac{4}{3}}} \\
\leqslant\|u\|_{L_{T}^{4} L_{x}^{4}}\left\|\int_{0}^{s} e^{-\left(s-s^{\prime}\right)}\right\| u\left(s^{\prime}\right)\left\|_{L^{4}}^{2} d s^{\prime}\right\|_{L_{T}^{2}} .
\end{gathered}
$$

We now apply the Paley-Littlewood-Sobolev inequality:

$$
\left\|\int_{0}^{s} e^{-\left(s-s^{\prime}\right)}\right\| u\left(s^{\prime}\right)\left\|_{L^{4}}^{2} d s^{\prime}\right\|_{L_{T}^{2}} \leqslant\left\|e^{-|s|} * \frac{1}{|s|^{2 \alpha}}\right\|_{L^{2}}\|u\|_{T}^{2} \lesssim\|u\|_{T}^{2}
$$

for $1+\frac{1}{2}=\frac{1}{p}+2 \alpha, p>1$, provided $e^{-|s|} \in L^{p}$. Since it is the case for all $p$, the estimate (18) holds for all $\alpha>\frac{1}{4}$.

Now, for $i=1$,

$$
\left\|\nabla \int_{0}^{s} e^{-\left(s-s^{\prime}\right)}\left|u\left(s^{\prime}\right)\right|^{2} u(s) d s^{\prime}\right\|_{L_{T}^{\frac{4}{3}} L_{x}^{\frac{4}{3}}} \leqslant\left\|\int_{0}^{s} e^{-\left(s-s^{\prime}\right)}\right\| u\left(s^{\prime}\right)\left\|_{L^{4}}^{2}\right\| \nabla u(s)\left\|_{L^{4}} d s^{\prime}\right\|_{L_{T}^{\frac{4}{3}}}
$$




$$
+\left\|\int_{0}^{s} e^{-\left(s-s^{\prime}\right)}\right\| u\left(s^{\prime}\right)\left\|_{L^{4}}\right\| \nabla u\left(s^{\prime}\right)\left\|_{L^{4}}\right\| \nabla u(s)\left\|_{L^{4}} d s^{\prime}\right\|_{L_{T}^{\frac{4}{3}}} .
$$

The first norm can be estimated exacly as in the previous situation.

We split the second norm in two parts:

On one hand,

$$
\begin{aligned}
& \left\|\int_{0}^{s} e^{-\left(s-s^{\prime}\right)}\right\| u\left(s^{\prime}\right)\left\|_{L^{4}}\right\| \nabla u\left(s^{\prime}\right)\left\|_{L^{4}}\right\| \nabla u(s)\left\|_{L^{4}} d s^{\prime}\right\|_{L_{T}^{\frac{4}{3}}} \\
\leqslant & \left\|\int_{0}^{s} e^{-\left(s-s^{\prime}\right)}\right\| u\left(s^{\prime}\right)\left\|_{L^{4}}\right\| \nabla u\left(s^{\prime}\right)\left\|_{L^{4}}\right\| \nabla u(s)\left\|_{L^{4}} d s^{\prime}\right\|_{L^{\frac{4}{3}}(0,1)} \\
+ & \left\|\int_{0}^{s} e^{-\left(s-s^{\prime}\right)}\right\| u\left(s^{\prime}\right)\left\|_{L^{4}}\right\| \nabla u\left(s^{\prime}\right)\left\|_{L^{4}}\right\| \nabla u(s)\left\|_{L^{4}} d s^{\prime}\right\|_{L_{T}^{\frac{4}{3}}(1, T)} .
\end{aligned}
$$

$$
\begin{aligned}
& \left\|\int_{0}^{s} e^{-\left(s-s^{\prime}\right)}\right\| u\left(s^{\prime}\right)\left\|_{L^{4}}\right\| \nabla u\left(s^{\prime}\right)\left\|_{L^{4}}\right\| \nabla u(s)\left\|_{L^{4}} d s^{\prime}\right\|_{L^{\frac{4}{3}}(0,1)} \\
& \leqslant\|\nabla u\|_{L_{T}^{4} L_{x}^{4}}\left\|\left(\int_{0}^{s} e^{-\frac{4}{3}\left(s-s^{\prime}\right)}\left\|u\left(s^{\prime}\right)\right\|_{L^{4}}^{\frac{4}{3}}\right)^{\frac{3}{4}}\right\| u(s)\left\|_{L^{4}}\right\|_{L^{\frac{4}{3}}(0,1)} \\
& \lesssim\|\nabla u\|_{L_{T}^{4} L_{x}^{4}}\|u\|_{L_{T}^{\infty} L_{x}^{4}}^{2} \lesssim\|\nabla u\|_{L_{T}^{4} L_{x}^{4}}\|u\|_{L_{T}^{\infty} H_{x}^{1}}^{2} .
\end{aligned}
$$

On the other hand,

$$
\begin{aligned}
& \left\|\int_{1}^{s} e^{-\left(s-s^{\prime}\right)}\right\| u\left(s^{\prime}\right)\left\|_{L^{4}}\right\| \nabla u\left(s^{\prime}\right)\left\|_{L^{4}}\right\| u(s)\left\|_{L^{4}} d s^{\prime}\right\|_{L^{\frac{4}{3}}(1, T)} \\
& \lesssim\|\nabla u\|_{L_{t}^{4} L_{x}^{4}}\|\| u(s)\left\|_{L^{4}}\left(\int_{1}^{s} e^{-\frac{4}{3}\left(s-s^{\prime}\right)}\left\|u\left(s^{\prime}\right)\right\|_{L^{4}}^{\frac{4}{3}}\right)^{\frac{3}{4}}\right\|_{L^{\frac{4}{3}}(1, T)} \\
& \quad \lesssim\|\nabla u\|_{L_{t}^{4} L_{x}^{4}}\left\|\left(e^{-\frac{4}{3}\left|s^{\prime}\right|} * \frac{1}{\left|s^{\prime}\right|^{\frac{4}{3} \alpha}}\right)^{\frac{3}{4}}(s) \frac{1}{s^{\alpha}}\right\|_{L^{\frac{4}{3}}(1, T)}
\end{aligned}
$$

and, by Hölder, for some $2<\delta<4$ to be chosen later,

$$
\left\|\left(e^{-\frac{4}{3}\left|s^{\prime}\right|} * \frac{1}{\left|s^{\prime}\right|^{\frac{4}{3} \alpha}}\right)^{\frac{3}{4}}(s) \frac{1}{s^{\alpha}}\right\|_{L^{\frac{4}{3}(1, T)}} \leqslant\left\|e^{-\frac{4}{3}\left|s^{\prime}\right|} * \frac{1}{\left|s^{\prime}\right|^{\frac{4}{3} \alpha}}\right\|_{L^{\frac{3 \delta}{30-4}}}\left\|\frac{1}{s^{\alpha}}\right\|_{L^{\delta}(1, T)}
$$

which is bounded for $\alpha>\max \left\{\frac{1}{\delta}, \frac{3}{4}-\frac{1}{\delta}\right\}$ and the lemma is proved by taking $\delta=\frac{8}{3}$.

\section{4 (Modified) scattering in dimension $d=1$}

In this section, we show Theorem 1.6, which states a modified scattering result in dimension $d=1$. As stated in the introduction, we deeply rely on the ideas in [13] which, in turn, derive from the works of Germain, Masmoudi and Shatah ([7]) concerning the space-time resonances method.

For $\left(u_{0}, v_{0}\right) \in \Sigma(\mathbb{R}) \times H^{1}(\mathbb{R})$ we consider the solution $(u, v)$ to the Cauchy problem (1). We begin by rewriting the integral version of (11) for initial data taken at $t=1$ :

$$
\begin{aligned}
u(x, t)=S( & -1) u(x, 1)+i \int_{1}^{t} S(t-s) u(s) v(s) d s \\
& =S(t-1) u(x, 1)+i \int_{1}^{t} S(t-s) u(s)\left(e^{-(s-1)} v(x, 1)+\int_{1}^{s} e^{-\left(s-s^{\prime}\right)}\left|u\left(s^{\prime}\right)\right|^{2} d s^{\prime}\right) d s
\end{aligned}
$$


or, putting $\left(u_{*}, v_{*}\right):=\left(e^{-i \frac{\Delta}{2}} u(1), e v(1)\right)$,

$$
u(x, t)=S(t) u_{*}(x)+i \int_{1}^{t} S(t-s) u(s)\left(e^{-s} v_{*}(x)+\int_{1}^{s} e^{-\left(s-s^{\prime}\right)}\left|u\left(s^{\prime}\right)\right|^{2} d s^{\prime}\right) d s .
$$

We now define the profile

$$
f(t):=S(-t) u(t)
$$

The proof of Theorem 1.6 relies essentially on the following Proposition (and its proof):

Proposition 4.1. Let $\left(u_{0}, v_{0}\right) \in \Sigma(\mathbb{R}) \times H^{1}(\mathbb{R})$ and $(u, v)$ the solution to (1) given by Theorem 3.2. Then, putting

$$
\|u\|_{Y_{T}}:=\left\|t^{\frac{1}{2}} u\right\|_{L_{T}^{\infty} L_{x}^{\infty}}+\left\|t^{-\alpha} u\right\|_{L_{T}^{\infty} H_{x}^{1}}+\left\|t^{-\alpha} x f\right\|_{L_{T}^{\infty} L_{x}^{2}}+\|u\|_{L_{T}^{\infty} L_{x}^{2}},
$$

for all $T>0$,

$$
\|u\|_{Y_{T}} \lesssim\left\|u_{*}\right\|_{\Sigma}+\left\|v_{*}\right\|_{H^{1}}\|u\|_{Y_{T}}+\|u\|_{Y_{T}}^{3} .
$$

Proof. By applying $S(-t)$ to (19) and taking the Fourier transform,

$$
\hat{f}(\xi)=\hat{u_{*}}+i \int_{1}^{t} e^{-i \frac{1}{2} \xi^{2} s} e^{-s} u \widehat{(s) v_{*}}(x)(\xi) d s+i \int_{1}^{t} \int_{1}^{s} e^{-i \frac{1}{2} \xi^{2} s} e^{-\left(s-s^{\prime}\right)} \mid u\left(\widehat{\left.s^{\prime}\right)\left.\right|^{2} u}(s)(\xi) d s^{\prime} d s,\right.
$$

that is

$$
\begin{aligned}
\hat{f}(\xi)= & \hat{u_{*}}+i \int_{1}^{t} e^{-i \frac{1}{2} \xi^{2} s} e^{-s} \int \hat{u}(\xi-\eta, s) \hat{v_{*}}(\eta) d \eta d s \\
& +i \int_{1}^{t} \int_{1}^{s} \iint e^{-i \frac{1}{2} \xi^{2} s} e^{-\left(s-s^{\prime}\right)} \hat{u}\left(\sigma, s^{\prime}\right) \overline{\hat{u}\left(\sigma-\eta, s^{\prime}\right)} \hat{u}(\xi-\eta, s) d \eta d \sigma d s^{\prime} d s \\
= & \hat{u_{*}}+i \int_{1}^{t} \int e^{-i \frac{1}{2}\left(\xi^{2}-(\xi-\eta)^{2}\right) s} e^{-s} \hat{f}(\xi-\eta, s) \hat{v_{*}}(\eta, s) d \eta d s \\
& +i \int_{1}^{t} \int_{1}^{s} \iint e^{i \phi} e^{-\left(s-s^{\prime}\right)} \hat{f}\left(\sigma, s^{\prime}\right) \overline{\hat{f}\left(\sigma-\eta, s^{\prime}\right)} \hat{f}(\xi-\eta, s) d \eta d \sigma d s^{\prime} d s,
\end{aligned}
$$

where

$$
\begin{aligned}
\phi=\phi(\xi, \eta, \sigma) & =-\frac{1}{2}\left(\xi^{2} s+\sigma^{2} s^{\prime}-(\sigma-\eta)^{2} s^{\prime}+(\xi-\eta)^{2} s\right) \\
& =\eta(\sigma-\xi) s+\left(\sigma \eta-\frac{1}{2} \eta^{2}\right)\left(s-s^{\prime}\right) .
\end{aligned}
$$

Finally, putting

$$
F(s, \xi, \sigma, \eta)=\int_{1}^{s} e^{i \frac{1}{2}\left(\eta^{2}-2 \eta \sigma\right)\left(s-s^{\prime}\right)} e^{-\left(s-s^{\prime}\right)} \hat{f}\left(\sigma, s^{\prime}\right) \overline{\hat{f}\left(\sigma-\eta, s^{\prime}\right)} \hat{f}(\xi-\eta, s) d s^{\prime},
$$

we obtain

$$
\begin{aligned}
\hat{f}(\xi)= & \hat{u_{*}}+i \int_{1}^{t} \int e^{i \frac{1}{2}\left(\eta^{2}-2 \xi \eta\right) s} e^{-s} \hat{f}(\xi-\eta, s) \hat{\psi_{*}}(\eta, s) d \eta d s \\
& +i \int_{1}^{t} \int_{1}^{s} \iint e^{i \eta(\sigma-\xi) s} F(s, \xi, \sigma, \eta) d s d \eta d \sigma,
\end{aligned}
$$

that is, by a change of variables in the last integral,

$$
\begin{aligned}
\hat{f}(\xi)= & \hat{u_{*}}+i \int_{1}^{t} \int e^{\frac{1}{2} i\left(\eta^{2}-2 \xi \eta\right) s} e^{-s} \hat{f}(\xi-\eta, s) \hat{\psi_{*}}(\eta, s) d \eta d s \\
& +i \int_{1}^{t} \int_{1}^{s} \iint e^{-i \eta \sigma s} F(s, \xi, \xi-\sigma, \eta) d s d \eta d \sigma .
\end{aligned}
$$


We now use these formulae to estimate the various norms appearing in $\|\cdot\|_{Y_{T}}$.

Estimate for $\left\|t^{-\alpha} \partial_{x} u\right\|_{L_{t}^{\infty} L_{x}^{2}}$ : From (19) we obtain

$$
\left\|\partial_{x} u\right\|_{L^{2}} \leqslant\left\|\partial_{x} u_{*}\right\|_{2}+\int_{1}^{t} e^{-s}\left\|\partial_{x}\left(u v_{*}\right)\right\|_{2} d s+\int_{1}^{t} \int_{1}^{s} e^{-\left(s-s^{\prime}\right)}\left\|\partial_{x}\left(u^{2}\left(s^{\prime}\right) u(s)\right)\right\|_{L^{2}} d s^{\prime} .
$$

Also,

$$
\begin{gathered}
\int_{1}^{t} e^{-s}\left\|\partial_{x}\left(u v_{*}\right)\right\|_{2} d s \leqslant \int_{1}^{t} e^{-s}\left(\left\|v_{*}\right\|_{\infty}\left\|\partial_{x} u\right\|_{2}+\left\|\partial_{x} v_{*}\right\|_{2}\|u\|_{\infty}\right) d s \\
\lesssim\left\|v_{*}\right\|_{H^{1}} \int_{1}^{t} e^{-s}\|u\|_{H^{1}} d s \lesssim\left\|v_{*}\right\|_{H^{1}}\|u\|_{Y_{T}} \int_{1}^{t} s^{\alpha} e^{-s} d s \lesssim\left\|v_{*}\right\|_{H^{1}}\|u\|_{Y_{T}} .
\end{gathered}
$$

Now, since

$$
\left\|\partial_{x}\left(u^{2}\left(s^{\prime}\right) u(s)\right)\right\|_{L^{2}} \lesssim\left\|u\left(s^{\prime}\right)\right\|_{\infty}^{2}\left\|\partial_{x} u(s)\right\|_{L^{2}}+\left\|u\left(s^{\prime}\right)\right\|_{\infty}\|u(s)\|_{\infty}\left\|\partial_{x} u\left(s^{\prime}\right)\right\|_{L^{2}},
$$

we obtain

$$
\begin{array}{r}
\int_{1}^{t} \int_{1}^{s} e^{-\left(s-s^{\prime}\right)}\left\|\partial_{x}\left(u^{2}\left(s^{\prime}\right) u(s)\right)\right\|_{L^{2}} d s^{\prime} d s \lesssim \int_{1}^{t}\left\|\partial_{x} u(s)\right\|_{L^{2}} \int_{1}^{s} e^{-\left(s-s^{\prime}\right)}\left\|u\left(s^{\prime}\right)\right\|_{\infty}^{2} d s^{\prime} d s \\
+\int_{1}^{t}\|u(s)\|_{\infty} \int_{1}^{s} e^{-\left(s-s^{\prime}\right)}\left\|u\left(s^{\prime}\right)\right\|_{\infty}\left\|\partial_{x} u\left(s^{\prime}\right)\right\|_{2} d s^{\prime} d s \\
\lesssim\left(\int_{1}^{t} s^{\alpha} \int_{1}^{s} e^{-\left(s-s^{\prime}\right)} s^{\prime-1} d s^{\prime} d s+\int_{1}^{t} s^{-\frac{1}{2}} \int_{1}^{s} e^{-\left(s-s^{\prime}\right)} s^{\prime \alpha-\frac{1}{2}} d s^{\prime} d s\right)\|u\|_{Y_{T}}^{2}
\end{array}
$$

In order to estimate these integrals, notice that for all $\beta \in \mathbb{R}$ and $\gamma>0$,

$$
\lim _{s \rightarrow \infty} \frac{\int_{1}^{s} \frac{e^{\gamma s^{\prime}}}{s^{\prime \beta}} d s^{\prime}}{\frac{e^{\gamma s}}{s^{\beta}}}=\frac{1}{\gamma}
$$

hence for all $\beta$ and for all $s \geqslant 1$,

$$
\int_{1}^{s} \frac{e^{\gamma s^{\prime}}}{s^{\prime \beta}} d s^{\prime} \lesssim \frac{e^{\gamma s}}{s^{\beta}}
$$

Hence,

$$
\int_{1}^{t} s^{\alpha} \int_{1}^{s} e^{-\left(s-s^{\prime}\right)} s^{\prime-1} d s^{\prime} d s+\int_{1}^{t} s^{-\frac{1}{2}} \int_{1}^{s} e^{-\left(s-s^{\prime}\right)} s^{\prime \alpha-\frac{1}{2}} d s^{\prime} d s \lesssim \int_{1}^{t} s^{\alpha-1} d s \lesssim t^{\alpha} .
$$

Finally,

$$
\left\|t^{-\alpha} \partial_{x} u\right\|_{L_{t}^{\infty} L_{x}^{2}} \lesssim\left\|\partial_{x} u_{*}\right\|_{L^{2}}+\left\|v_{*}\right\|_{H^{1}}\|u\|_{Y_{T}}+\|u\|_{Y_{T}}^{3} .
$$

Estimate for $\left\|t^{-\alpha} x f\right\|_{L_{t}^{\infty} L_{x}^{2}}$ From (21),

$$
\begin{aligned}
\|x f\|_{L^{2}}=\left\|\partial_{\xi} \hat{f}\right\|_{L^{2}} \lesssim\left\|\partial_{\xi} \widehat{u *}\right\|_{L^{2}} & \\
& +\left\|\int_{1}^{t} \int \partial_{\xi}\left(e^{i \frac{1}{2}\left(\eta^{2}-2 \xi \eta\right) s} e^{-s} \hat{f}(\xi-\eta, s) \hat{v_{*}}(\eta)\right) d \eta d s\right\|_{L^{2}} \\
& +\left\|\int_{1}^{t} \int_{1}^{s} \iint \partial_{\xi}\left(e^{-i \eta \sigma s} F(s, \xi, \xi-\sigma, \eta)\right) d s d \eta d \sigma\right\|_{L^{2}}
\end{aligned}
$$




$$
\begin{gathered}
\lesssim\left\|x u_{*}\right\|_{L^{2}}+\left\|\int_{1}^{t} \int\left(e^{i \frac{1}{2}\left(|\xi-\eta|^{2}-\xi^{2}\right) s} e^{-s} \hat{f}(\xi-\eta, s) \eta \hat{v_{*}}(\eta)\right) d \eta d s\right\|_{L^{2}} \\
+\left\|\int_{1}^{t} \int\left(e^{i \frac{1}{2}\left(|\xi-\eta|^{2}-\xi^{2}\right) s} e^{-s} \widehat{[x f]}(\xi-\eta, s) \hat{v_{*}}(\eta)\right) d \eta d s\right\|_{L^{2}} \\
\quad+\left\|\int_{1}^{t} \int_{1}^{s} \iint\left(e^{-i \eta \sigma s} \partial_{\xi} F(s, \xi, \xi-\sigma, \eta)\right) d s d \eta d \sigma\right\|_{L^{2}} \\
\lesssim\left\|x u_{*}\right\|_{L^{2}}+I+I I+I I I
\end{gathered}
$$

We proceed with the estimation of the three integrals in the right-hand-side of (23):

$$
\begin{aligned}
& I \lesssim\left\|\left(\int_{1}^{t} e^{-s} S(-s) \int \widehat{S(s) f}(\xi-\eta, s) \widehat{\partial_{x} v_{*}}(\eta, s) d \eta d s\right)\right\|_{L^{2}} \\
& \lesssim \int_{0}^{t} e^{-s}\left\|\mathcal{F}\left(u(s) \partial_{x} v_{*}\right)\right\|_{L^{2}} d s \lesssim\left\|\partial_{x} v_{*}\right\|_{L^{2}} \int_{0}^{t} e^{-s}\|u(s)\|_{L^{\infty}} d s \\
& \lesssim\left\|\partial_{x} v_{*}\right\|_{L^{2}}\|u\|_{Y_{T}} \int_{0}^{t} e^{-s} s^{\frac{1}{2}} d s \lesssim\left\|\partial_{x} v_{*}\right\|_{L^{2}}\|u\|_{Y_{T}} .
\end{aligned}
$$

Similarly,

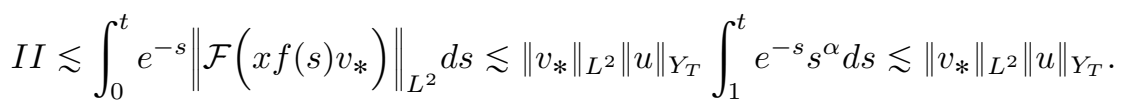

We now estimate $I I I$. First, notice that

$$
\begin{aligned}
& \partial_{\xi} F(s, \xi, \xi-\sigma, \eta)= \\
& \quad 2 i \int_{1}^{s}\left(s^{\prime}-s\right) \eta e^{i \frac{1}{2}\left(\eta^{2}-2 \eta(\xi-\sigma)\right)\left(s-s^{\prime}\right)} e^{-\left(s-s^{\prime}\right)} \hat{f}(\xi-\eta, s) \overline{\hat{f}\left(\xi-\eta-\sigma, s^{\prime}\right)} \hat{f}\left(\xi-\sigma, s^{\prime}\right) d s^{\prime} \\
& \quad+\int_{1}^{s} e^{i \frac{1}{2}\left(\eta^{2}-2 \eta(\xi-\sigma)\right)\left(s-s^{\prime}\right)} e^{-\left(s-s^{\prime}\right)} \partial_{\xi}\left(\hat{f}(\xi-\eta, s) \overline{\hat{f}\left(\xi-\eta-\sigma, s^{\prime}\right)} \hat{f}\left(\xi-\sigma, s^{\prime}\right)\right) d s^{\prime}
\end{aligned}
$$

Writing $\eta=(\xi-\sigma)-(\xi-\sigma-\eta)$,

$$
\begin{aligned}
& \partial_{\xi} F(s, \xi, \xi-\sigma, \eta)= \\
& -2 \int_{1}^{s}\left(s^{\prime}-s\right) e^{i \frac{1}{2}\left(\eta^{2}-2 \eta(\xi-\sigma)\right)\left(s-s^{\prime}\right)} e^{-\left(s-s^{\prime}\right)} \hat{f}(\xi-\eta, s) \overline{\hat{f}\left(\xi-\eta-\sigma, s^{\prime}\right)} \widehat{\partial_{x} f}\left(\xi-\sigma, s^{\prime}\right) d s^{\prime} \\
& -2 \int_{1}^{s}\left(s^{\prime}-s\right) e^{i \frac{1}{2}\left(\eta^{2}-2 \eta(\xi-\sigma)\right)\left(s-s^{\prime}\right)} e^{-\left(s-s^{\prime}\right)} \hat{f}(\xi-\eta, s) \widehat{\widehat{\partial_{x} f}\left(\xi-\eta-\sigma, s^{\prime}\right)} \hat{f}\left(\xi-\sigma, s^{\prime}\right) d s^{\prime} \\
& \quad+\int_{1}^{s} e^{i \frac{1}{2}\left(\eta^{2}-2 \eta(\xi-\sigma)\right)\left(s-s^{\prime}\right)} e^{-\left(s-s^{\prime}\right)} \partial_{\xi}\left(\hat{f}(\xi-\eta, s) \overline{\left.\hat{f}\left(\xi-\eta-\sigma, s^{\prime}\right) \hat{f}\left(\xi-\sigma, s^{\prime}\right)\right) d s^{\prime}}\right. \\
& :=G_{1}(s, \xi, \eta, \sigma)+G_{2}(s, \xi, \eta, \sigma)+G_{3}(s, \xi, \eta, \sigma) .
\end{aligned}
$$

Now,

$$
\begin{aligned}
\left\|\int_{1}^{t} \iint e^{-i \eta \sigma s} G_{1}(s, \xi, \eta, \sigma) d \eta d \sigma d s\right\|_{L^{2}} & \lesssim \| \int_{1}^{t} \int_{1}^{s}\left(s^{\prime}-s\right) e^{-\left(s-s^{\prime}\right)} \\
& \iint e^{i \frac{1}{2}\left(\eta^{2}-2 \eta(\xi-\sigma)\right)\left(s-s^{\prime}\right)} e^{-i \eta \sigma s} \hat{f}(\xi-\eta, s) \widehat{\widehat{\partial_{x} f}\left(\xi-\eta-\sigma, s^{\prime}\right)} \widehat{\partial_{x} f}\left(\xi-\sigma, s^{\prime}\right) d \eta d \sigma d s^{\prime} d s \|_{L^{2}} .
\end{aligned}
$$


By redistributing the phase term, that is, observing that

$$
\left(\eta^{2}-2 \eta(\xi-\sigma)\right)\left(s-s^{\prime}\right)-2 \sigma \eta s=-\xi^{2} s+(\xi-\eta)^{2} s-(\xi-\eta-\sigma)^{2} s^{\prime}+(\xi-\sigma)^{2} s^{\prime},
$$

we obtain

$$
\begin{gathered}
\left\|\int_{1}^{t} \iint e^{-2 i \eta \sigma s} G_{1}(s, \xi, \eta, \sigma) d \eta d \sigma d s\right\|_{L^{2}} \\
\lesssim\left\|\mathcal{F} \int_{1}^{t} \int_{1}^{s}\left(s^{\prime}-s\right) e^{-\left(s-s^{\prime}\right)} S(-s)\left[S(s) f(s) \overline{S\left(s^{\prime}\right) f\left(s^{\prime}\right)} S\left(s^{\prime}\right) \partial_{x} f\left(s^{\prime}\right)\right] d s^{\prime} d s\right\|_{L^{2}} \\
\lesssim \int_{1}^{t}\|u(s)\|_{L^{\infty}} \int_{1}^{s}\left(s-s^{\prime}\right) e^{-\left(s-s^{\prime}\right)}\left\|u\left(s^{\prime}\right)\right\|_{L^{\infty}}\left\|\partial_{x} u\left(s^{\prime}\right)\right\|_{L^{2}} d s^{\prime} d s \\
\lesssim\|u\|_{Y_{T}}^{3} \int_{1}^{t} s^{-\frac{1}{2}} \int_{1}^{s} e^{-\frac{1}{2}\left(s-s^{\prime}\right)} s^{\prime-\frac{1}{2}+\alpha} d s^{\prime} d s \lesssim\|u\|_{Y_{T}}^{3} \int_{1}^{t} s^{\alpha-1} d s \lesssim t^{\alpha}\|u\|_{Y_{T}}^{3}
\end{gathered}
$$

by (22), and, analogously,

$$
\left\|\int_{1}^{t} \iint e^{-i \eta \sigma s} G_{2}(s, \xi, \eta, \sigma) d \eta d \sigma d s\right\|_{L^{2}} \lesssim t^{\alpha}\|u\|_{Y_{T}}^{3} .
$$

With respect to the third integral, similar computations yield

$$
\begin{gathered}
\left\|\int_{1}^{t} \iint e^{-i \eta \sigma s} G_{3}(s, \xi, \eta, \sigma) d \eta d \sigma d s\right\|_{L^{2}} \\
\quad \lesssim \int_{1}^{t} \int_{1}^{s} e^{-\left(s-s^{\prime}\right)}\|x f(s)\|_{L^{2}}\left\|u\left(s^{\prime}\right)\right\|_{L^{\infty}}^{2} d s^{\prime} d s \\
\quad+\int_{1}^{t} \int_{1}^{s} e^{-\left(s-s^{\prime}\right)}\left\|x f\left(s^{\prime}\right)\right\|_{L^{2}}\|u(s)\|_{L^{\infty}}\left\|u\left(s^{\prime}\right)\right\|_{L^{\infty}} d s^{\prime} d s \\
\lesssim\|u\|_{Y_{T}}^{3}\left(\int_{1}^{t} \int_{1}^{s} e^{-\left(s-s^{\prime}\right)} s^{\prime-1} s^{\alpha} d s^{\prime} d s+\int_{1}^{t} \int_{1}^{s} e^{-\left(s-s^{\prime}\right)} s^{\prime \alpha-\frac{1}{2}} s^{-\frac{1}{2}} d s^{\prime} d s\right) \lesssim\|u\|_{Y_{T}}^{3} t^{\alpha} .
\end{gathered}
$$

Finally, $I I I \lesssim\|u\|_{Y_{T}}^{3} t^{\alpha}$ and we obtain

$$
\|x f\|_{L^{2}} \lesssim\left\|x u_{*}\right\|_{L^{2}}+\left\|v_{*}\right\|_{H^{1}}\|u\|_{Y_{T}}+\|u\|_{Y_{T}}^{3} t^{\alpha}
$$

that is

$$
\left\|t^{-\alpha} x f\right\|_{L_{T}^{\infty} L_{x}^{2}} \lesssim\left\|\partial_{x} u_{*}\right\|_{L^{2}}+\left\|v_{*}\right\|_{H^{1}}\|u\|_{Y_{T}}+\|u\|_{Y_{T}}^{3} .
$$

Estimate for $\left\|t^{\frac{1}{2}} u\right\|_{L_{t}^{\infty} L_{x}^{\infty}}$ : From (20), we write

$$
\begin{aligned}
\hat{f}(\xi)= & \hat{u_{*}}+i \int_{1}^{t} \int e^{-i \frac{1}{2}\left(\xi^{2}-(\xi-\eta)^{2}\right) s} e^{-s} \hat{f}(\xi-\eta, s) \hat{v_{*}}(\eta, s) d \eta d s \\
& +i \int_{1}^{t} \int_{1}^{s} \iint e^{i \phi} e^{-\left(s-s^{\prime}\right)} \hat{f}\left(\sigma, s^{\prime}\right) \bar{f}\left(\sigma-\eta, s^{\prime}\right) \hat{f}(\xi-\eta, s) d \eta d \sigma d s^{\prime} d s \\
= & \hat{u_{*}}+i \int_{1}^{t} R_{1}(\xi, s) d s+i \int_{1}^{t} \int_{1}^{s} \iint e^{i \phi} G\left(s^{\prime}, s, \xi, \eta, \sigma\right) d \eta d \sigma d s^{\prime} d s,
\end{aligned}
$$

where

$$
G\left(s^{\prime}, s, \xi, \eta, \sigma\right)=e^{-\left(s-s^{\prime}\right)} \hat{f}\left(\sigma, s^{\prime}\right) \overline{\hat{f}\left(\sigma-\eta, s^{\prime}\right)} \hat{f}(\xi-\eta, s),
$$




$$
R_{1}(\xi, s)=\int e^{-i \frac{1}{2}\left(\xi^{2}-(\xi-\eta)^{2}\right) s} e^{-s} \hat{f}(\xi-\eta, s) \hat{v_{*}}(\eta, s) d \eta
$$

and

$$
\phi=-\xi \eta s+\frac{1}{2} \eta^{2} s-\frac{1}{2} \eta^{2} s^{\prime}+\eta \sigma s^{\prime}=-\xi \eta s+\eta \tilde{\sigma} s,
$$

with $\tilde{\sigma}=\frac{1}{2 s}\left(\eta\left(s-s^{\prime}\right)+2 \sigma s^{\prime}\right)$. By performing the change of variables $\sigma \mapsto \tilde{\sigma}$, we obtain

$$
\begin{gathered}
\int_{1}^{t} \int_{1}^{s} \iint e^{i \phi} G(s, \xi, \eta, \sigma) d \eta d \sigma d s^{\prime} d s \\
=\int_{1}^{t} \int_{1}^{s} \iint e^{i \eta \xi s} e^{-i \eta \sigma s} \frac{s}{s^{\prime}} G\left(s^{\prime}, s, \xi, \eta, \sigma \frac{s}{s^{\prime}}-\eta \frac{s-s^{\prime}}{2 s^{\prime}}\right) d \eta d \sigma d s^{\prime} d s \\
=\int_{1}^{t} \int_{1}^{s} \iint \frac{s}{s^{\prime}} \mathcal{F}_{\eta, \sigma}\left(e^{i \eta \xi s} e^{-i \eta \sigma s}\right) \mathcal{F}_{\eta, \sigma}^{-1}\left(G\left(s^{\prime}, s, \xi, \eta, \sigma \frac{s}{s^{\prime}}-\eta \frac{s-s^{\prime}}{2 s^{\prime}}\right)\right) d \eta^{\prime} d \sigma^{\prime} d s^{\prime} d s
\end{gathered}
$$

by Plancherel. Furthermore,

$$
\mathcal{F}_{\eta, \sigma}\left(e^{i \eta \xi s} e^{-i \eta \sigma s}\right)=\mathcal{F}_{\eta, \sigma}\left(e^{-i \eta \sigma s}\right)\left(\eta^{\prime}-\xi s, \sigma^{\prime}\right)=\frac{1}{2 s} e^{i \frac{1}{2} \frac{i \eta^{\prime} \sigma^{\prime}}{2 s}} e^{-i \frac{1}{2} \xi \sigma^{\prime}} .
$$

On the other hand,

$$
\begin{aligned}
& \mathcal{F}_{\sigma}^{-1}\left(G\left(s^{\prime}, s, \xi, \eta, \sigma \frac{s}{s^{\prime}}-\eta \frac{s-s^{\prime}}{2 s^{\prime}}\right)\right) \\
= & \left.\left.\left.e^{-\left(s-s^{\prime}\right)} \hat{f}(\xi-\eta, s) \mathcal{F}_{\sigma}^{-1}\left(\overline{\hat{f}\left(\frac{s}{s^{\prime}}\left(\sigma-\eta \frac{s+s^{\prime}}{4 s}\right)\right.}, s^{\prime}\right) \hat{f}\left(\frac{s}{s^{\prime}}\left(\sigma-\eta \frac{s+s^{\prime}}{2 s}+\eta \frac{s^{\prime}}{s}\right)\right), s^{\prime}\right)\right)\right) \\
= & \left.e^{-\left(s-s^{\prime}\right)} \hat{f}(\xi-\eta, s) e^{i \sigma^{\prime} \eta \frac{s+s^{\prime}}{4 s}} \mathcal{F}_{\sigma}^{-1}\left(\overline{\hat{f}\left(\frac{s}{s^{\prime}} \sigma, s^{\prime}\right)} \hat{f}\left(\frac{s}{s^{\prime}} \sigma+\eta, s^{\prime}\right)\right)\right) \\
= & \frac{s^{\prime}}{s} \hat{f}(\xi-\eta, s) e^{i \sigma^{\prime} \eta \frac{s+s^{\prime}}{4 s}} \mathcal{F}_{\sigma}^{-1}\left(\overline{\hat{f}}\left(\sigma, s^{\prime}\right) \hat{f}\left(\sigma+\eta, s^{\prime}\right)\right)\left(\frac{s^{\prime} \sigma^{\prime}}{s}, \eta^{\prime}\right) \\
= & \frac{s^{\prime}}{s} \hat{f}(\xi-\eta, s) e^{i \sigma^{\prime} \eta \frac{s+s^{\prime}}{4 s}}\left[\frac{\bar{f}}{*} *_{\sigma} e^{-i \sigma \eta} f\right]\left(\frac{s^{\prime} \sigma^{\prime}}{s}\right) \\
= & \frac{s^{\prime}}{s} \hat{f}(\xi-\eta, s) e^{i \sigma^{\prime} \eta \frac{s+s^{\prime}}{4 s}} \int e^{-i \eta x} f\left(x, s^{\prime}\right) \bar{f}\left(x-\frac{s^{\prime} \sigma^{\prime}}{s}, s^{\prime}\right) d x,
\end{aligned}
$$

where $\check{g}(x)=g(-x)$. Also,

$$
\begin{aligned}
\mathcal{F}_{\eta}^{-1}\left[\hat{f}(\xi-\eta, s) e^{i \frac{1}{2} \sigma^{\prime} \eta \frac{s+s^{\prime}}{2 s}} e^{-i \eta x}\right]\left(\eta^{\prime}\right)=\mathcal{F}_{\eta}^{-1}[\hat{f}(\xi-\eta, s)]\left(\eta^{\prime}+\sigma^{\prime} \frac{s+s^{\prime}}{2 s}-x\right) \\
=\left[e^{i \eta \xi} \tilde{f}\right]\left(\eta^{\prime}+\sigma^{\prime} \frac{s+s^{\prime}}{2 s}-x, s\right)=e^{\frac{1}{2} i\left(\eta^{\prime}+\sigma^{\prime} \frac{s+s^{\prime}}{2 s}-x\right) \xi} f\left(x-\sigma^{\prime} \frac{s+s^{\prime}}{2 s}-\eta^{\prime}, s\right)
\end{aligned}
$$

Finally,

$$
\begin{aligned}
& \mathcal{F}_{\eta, \sigma}^{-1}\left(G\left(s^{\prime}, s, \xi, \eta, \sigma \frac{s}{s^{\prime}}-\eta \frac{s-s^{\prime}}{2 s^{\prime}}\right)\right) \\
&=e^{-\left(s-s^{\prime}\right)} \frac{s^{\prime}}{s} \int e^{i \frac{1}{2}\left(\eta^{\prime}+\sigma^{\prime} \frac{s+s^{\prime}}{2 s}-x\right) \xi} f\left(x-\sigma^{\prime} \frac{s+s^{\prime}}{2 s}-\eta^{\prime}, s\right) f\left(x, s^{\prime}\right) \bar{f}\left(x-\frac{s^{\prime} \sigma^{\prime}}{s}, s^{\prime}\right) d x
\end{aligned}
$$


By (24),

$$
\begin{gathered}
\int_{1}^{t} \int_{1}^{s} \iint e^{i \phi} G(s, \xi, \eta, \sigma) d \eta d \sigma d s^{\prime} d s \\
=\int_{1}^{t} \int_{1}^{s} \iint \frac{1}{2 s^{\prime}} e^{i \frac{i \eta^{\prime} \sigma^{\prime}}{4 s}} e^{-\frac{1}{2} i \xi \sigma^{\prime}} \mathcal{F}_{\eta, \sigma}^{-1}\left(G\left(s^{\prime}, s, \xi, \eta, \sigma \frac{s}{s^{\prime}}-\eta \frac{s-s^{\prime}}{2 s^{\prime}}\right)\right) d \eta^{\prime} d \sigma^{\prime} d s^{\prime} d s \\
=\int_{1}^{t} \int_{1}^{s} \iint \frac{1}{2 s^{\prime}} e^{-\frac{1}{2} i \xi \sigma^{\prime}} \mathcal{F}_{\eta, \sigma}^{-1}\left(G\left(s^{\prime}, s, \xi, \eta, \sigma \frac{s}{s^{\prime}}-\eta \frac{s-s^{\prime}}{2 s^{\prime}}\right)\right) d \eta^{\prime} d \sigma^{\prime} d s^{\prime} d s \\
+\int_{1}^{t} \int_{1}^{s} \iint \frac{1}{2 s^{\prime}}\left(e^{i \frac{i \eta^{\prime} \sigma^{\prime}}{2 s}}-1\right) e^{-\frac{1}{2} i \xi \sigma^{\prime}} \mathcal{F}_{\eta, \sigma}^{-1}\left(G\left(s^{\prime}, s, \xi, \eta, \sigma \frac{s}{s^{\prime}}-\eta \frac{s-s^{\prime}}{2 s^{\prime}}\right)\right) d \eta^{\prime} d \sigma^{\prime} d s^{\prime} d s \\
=\int_{1}^{t} \int_{1}^{s} \frac{1}{2 s^{\prime}} G\left(s^{\prime}, s, \xi, 0, \xi \frac{s}{s^{\prime}}\right) d s^{\prime} d s+\int_{1}^{t} R_{2}(\xi, s) d s \\
=\int_{1}^{t}\left(\int_{1}^{s} \frac{1}{2 s^{\prime}} e^{-\left(s-s^{\prime}\right)}\left|\hat{f}\left(\frac{s}{s^{\prime}} \xi, s^{\prime}\right)\right|^{2} d s^{\prime}\right) \hat{f}(\xi, s) d s+\int_{1}^{t} R_{2}(\xi, s) d s
\end{gathered}
$$

where

$$
R_{2}(\xi, s)=\int_{1}^{s} \iint \frac{1}{2 s^{\prime}}\left(e^{i \frac{i \eta^{\prime} \sigma^{\prime}}{4 s}}-1\right) e^{-\frac{1}{2} i \xi \sigma^{\prime}} \mathcal{F}_{\eta, \sigma}^{-1}\left(G\left(s^{\prime}, s, \xi, \eta, \sigma \frac{s}{s^{\prime}}-\eta \frac{s-s^{\prime}}{2 s^{\prime}}\right)\right) d \eta^{\prime} d \sigma^{\prime} d s^{\prime}
$$

Furthermore,

$$
\left|R_{1}(\xi, s)\right| \leqslant\|u\|_{Y_{T}}\left\|v_{*}\right\|_{L^{2}} e^{-s}
$$

and

$$
\begin{aligned}
& \left|R_{2}(\xi, s)\right| \lesssim \\
& \quad \int_{1}^{s} \frac{1}{s} e^{-\left(s-s^{\prime}\right)}\left|\sin \left(\frac{\eta^{\prime} \sigma^{\prime}}{4 s^{\prime}}\right)\right|\left|f\left(x-\sigma^{\prime} \frac{s+s^{\prime}}{2 s}-\eta^{\prime}, s\right)\right|\left|f\left(x, s^{\prime}\right)\right|\left|f\left(x-\frac{s^{\prime} \sigma^{\prime}}{s}, s^{\prime}\right)\right| d s^{\prime} d \eta^{\prime} d \sigma^{\prime} d x \\
& \quad \lesssim \int_{1}^{s} \frac{1}{s s^{\prime \delta}} e^{-\left(s-s^{\prime}\right)}\left|\eta^{\prime}\right|^{\delta}\left|\sigma^{\prime}\right|^{\delta}\left|f\left(x-\sigma^{\prime} \frac{s+s^{\prime}}{2 s}-\eta^{\prime}, s\right)\right|\left|f\left(x, s^{\prime}\right)\right|\left|f\left(x-\frac{s^{\prime} \sigma^{\prime}}{s}, s^{\prime}\right)\right| d s^{\prime} d \eta^{\prime} d \sigma^{\prime} d x
\end{aligned}
$$

for all $0<\delta<\frac{1}{2}$. Also, observe that

$$
\begin{aligned}
\left|\eta^{\prime}\right|^{\delta} \leqslant\left|x-\sigma^{\prime} \frac{s+s^{\prime}}{2 s}-\eta^{\prime}\right|^{\delta}+\left|x-\sigma^{\prime} \frac{s+s^{\prime}}{2 s}\right|^{\delta} \leqslant & \left|x-\sigma^{\prime} \frac{s+s^{\prime}}{2 s}-\eta^{\prime}\right|^{\delta} \\
& +\left(\frac{s+s^{\prime}}{2 s^{\prime}}\right)^{\delta}\left|x-\frac{s^{\prime}}{s} \sigma^{\prime}\right|^{\delta}+\left(1+\frac{s+s^{\prime}}{2 s^{\prime}}\right)^{\delta}|x|^{\delta}
\end{aligned}
$$

and

$$
\left|\sigma^{\prime}\right|^{\delta} \leqslant\left(\frac{s}{s^{\prime}}\right)^{\delta}\left|x-\frac{s^{\prime}}{s} \sigma^{\prime}\right|^{\delta}+\left(\frac{s}{s^{\prime}}\right)^{\delta}|x|^{\delta} .
$$

We now have six terms to estimate. We will only treat one of the most significant, the remaining follow by similar computations:

$$
\begin{aligned}
& \int_{1}^{s} \frac{1}{s^{1-2 \delta} s^{\prime 3 \delta}} e^{-\left(s-s^{\prime}\right)}|x|^{2 \delta}\left|f\left(x-\sigma^{\prime} \frac{s+s^{\prime}}{2 s}-\eta^{\prime}, s\right)\right|\left|f\left(x, s^{\prime}\right)\right|\left|f\left(x-\frac{s^{\prime} \sigma^{\prime}}{s}, s^{\prime}\right)\right| d s^{\prime} d x d \eta^{\prime} d \sigma^{\prime} \\
& \lesssim\|u\|_{Y_{T}}^{3} \int_{1}^{s} e^{-\left(s-s^{\prime}\right)} s^{2 \alpha-3 \delta-1} s^{2 \delta+\alpha} d s^{\prime} \lesssim\|u\|_{Y_{T}}^{3} s^{3 \alpha-\delta-1}
\end{aligned}
$$


Hence, taking the time derivative of (4),

$$
\begin{gathered}
\partial_{t} \hat{f}(\xi, t)=i\left(\int_{1}^{t} \frac{1}{2 s^{\prime}} e^{-\left(t-s^{\prime}\right)}\left|\hat{f}\left(\frac{s}{s^{\prime}} \xi, s^{\prime}\right)\right|^{2} d s^{\prime}\right) \hat{f}(\xi, t)+\int_{1}^{t} R(\xi, s), \\
|R(\xi, s)| \leqslant\left|R_{1}(\xi, s)+R_{2}(\xi, s)\right| \lesssim\left\|v_{*}\right\|_{L^{2}}\|u\|_{Y_{T}} e^{-s}+\|u\|_{Y_{T}}^{3} s^{3 \alpha-\delta-1} .
\end{gathered}
$$

We can now complete the proof of the $L^{\infty}$ estimate: fix $\alpha, \beta, \delta>0$ so that $3 \alpha<\delta<1 / 2$ and $\alpha<\beta<1 / 4$ and write

$$
\Psi(\xi, t)=\int_{1}^{t} \int_{1}^{s} \frac{1}{2 s^{\prime}} e^{-\left(s-s^{\prime}\right)}\left|\hat{f}\left(\frac{s}{s^{\prime}} \xi, s^{\prime}\right)\right|^{2} d s^{\prime} d s \quad \text { and } \quad \hat{w}(\xi, t)=\hat{f}(\xi, t) e^{i \Psi(\xi, t)}
$$

Using the integrating factor $e^{i \Psi(\xi, t)}$ in (25),

$$
\hat{w}_{t}=e^{i \Psi(\xi, t)} R(t \xi), \quad t>0 .
$$

Therefore

$$
\begin{gathered}
|\hat{f}(\xi, t)|=|\hat{w}(\xi, t)| \leqslant|\hat{w}(\xi, 0)|+\int_{1}^{t}\left|\partial_{t} \hat{w}(\xi, s)\right| d s \leqslant|\hat{f}(\xi, 0)|+\int_{1}^{t}|R(\xi, s)| d s \\
\lesssim\left\|x u_{*}\right\|_{L^{2}}+\left\|v_{*}\right\|_{L^{2}}\|u\|_{Y_{T}} e^{-t}+\|u\|_{Y_{T}}^{3} t^{3 \alpha-\delta} .
\end{gathered}
$$

Using Lemma 2.2 in [11,

$$
\|u(t)\|_{L^{\infty}} \leqslant \frac{1}{t^{\frac{1}{2}}}\|\hat{f}\|_{L^{\infty}}+\frac{1}{t^{\frac{1}{2}+\beta}}\|x f\|_{L^{2}} \lesssim \frac{1}{t^{\frac{1}{2}}}\left(\left\|x u_{*}\right\|_{L^{2}}+\left\|v_{*}\right\|_{L^{2}}\|u\|_{Y_{T}}+\|u\|_{Y_{T}}^{3}\right)
$$

and

$$
\left\|t^{\frac{1}{2}} u\right\|_{L_{t}^{\infty} L_{x}^{\infty}} \lesssim\left\|x u_{*}\right\|_{L^{2}}+\left\|v_{*}\right\|_{L^{2}}\|u\|_{Y_{T}}+\|u\|_{Y_{T}}^{3}
$$

which concludes the proof of Proposition 4.1

Proof of Theorem 1.6. From Proposition 4.1 it is straightforward, once again by an obstruction argument, to show that $\|u\|_{Y_{T}}$ remains uniformly bounded as $t \rightarrow+\infty$ for small initial data. The decay rate of $\|u\|_{L^{\infty}}$ and the scattering result are then immediate consequences of the proof of the $L^{\infty}$ estimate (26).

Acknowledgements Simão Correia was partially supported by Fundação para a Ciência e Tecnologia, through the grant SFRH/BD/96399/2013 and through contract UID/MAT/04561/2013. Filipe Oliveira was partially supported by the Project CEMAPRE - UID/ MULTI/00491/2013 financed by FCT/MCTES through national funds.

\section{References}

[1] Brigitte Bidégaray. On the Cauchy problem for some systems occurring in nonlinear optics. Adv. Differential Equations, 3(3):473-496, 1998.

[2] Brigitte Bidégaray. The Cauchy problem for Schrödinger-Debye equations. Math. Models Methods Appl. Sci., 10(3):307-315, 2000.

[3] Thierry Cazenave and Fred B. Weissler. Rapidly decaying solutions of the nonlinear Schrödinger equation. Comm. Math. Phys., 147(1):75-100, 1992. 
[4] A. J. Corcho and F. Linares. Well-posedness for the Schrödinger-Debye equation. In Partial differential equations and inverse problems, volume 362 of Contemp. Math., pages 113-131. Amer. Math. Soc., Providence, RI, 2004.

[5] Adán J. Corcho, Filipe Oliveira, and Jorge Drumond Silva. Local and global well-posedness for the critical Schrödinger-Debye system. Proc. Amer. Math. Soc., 141(10):3485-3499, 2013.

[6] Adán J. Corcho and Jorge Drumond Silva. On the unboundedness of higher regularity Sobolev norms of solutions for the critical Schrödinger-Debye system with vanishing relaxation delay. Nonlinearity, 30(1):300-328, 2017.

[7] P. Germain, N. Masmoudi, and J. Shatah. Global solutions for 2D quadratic Schrödinger equations. J. Math. Pures Appl. (9), 97(5):505-543, 2012.

[8] J. Ginibre and G. Velo. On a class of nonlinear Schrödinger equations. II. Scattering theory, general case. J. Funct. Anal., 32(1):33-71, 1979.

[9] J. Ginibre and G. Velo. Scattering theory in the energy space for a class of nonlinear Schrödinger equations. J. Math. Pures Appl. (9), 64(4):363-401, 1985.

[10] J. Ginibre and G. Velo. Time decay of finite energy solutions of the nonlinear Klein-Gordon and Schrödinger equations. Ann. Inst. H. Poincaré Phys. Théor., 43(4):399-442, 1985.

[11] Nakao Hayashi and Pavel I. Naumkin. Asymptotics for large time of solutions to the nonlinear Schrödinger and Hartree equations. Amer. J. Math., 120(2):369-389, 1998.

[12] Nakao Hayashi, Pavel I. Naumkin, and Tohru Ozawa. Scattering theory for the Hartree equation. SIAM J. Math. Anal., 29(5):1256-1267, 1998.

[13] Jun Kato and Fabio Pusateri. A new proof of long-range scattering for critical nonlinear Schrödinger equations. Differential Integral Equations, 24(9-10):923-940, 2011.

[14] Tosio Kato. On nonlinear Schrödinger equations. II. $H^{s}$-solutions and unconditional wellposedness. J. Anal. Math., 67:281-306, 1995.

[15] A. C. Newell and J. V. Moloney. Nonlinear optics. Addison-Wesley, 1992.

[16] Yoshio Tsutsumi. Scattering problem for nonlinear Schrödinger equations. Ann. Inst. H. Poincaré Phys. Théor., 43(3):321-347, 1985.

SiMÃo CORREIA

CMAF-CIO and FCUL

Universidade de Lisboa

Campo Grande, Edifício C6, Piso 2, 1749-016 Lisboa, Portugal

sfcorreia@fc.ul.pt

Filipe Oliveira

Mathematics Department and CEMAPRE

ISEG, Universidade de Lisboa

Rua do Quelhas 6, 1200-781 Lisboa, Portugal

foliveira@iseg.ulisboa.pt 